\title{
Rpi-amr3 confers resistance to multiple Phytophthora species by recognizing a conserved RXLR effector
}

Xiao Lin ${ }^{1}$, Andrea Olave-Achury ${ }^{1}$, Robert Heal ${ }^{1}$, Kamil Witek ${ }^{1}$, Hari S. Karki ${ }^{1 \#}$, Tianqiao Song ${ }^{1 \#}$, Chih-hang $\mathrm{Wu}^{1 \#}$, Hiroaki Adachi ${ }^{1 \#}$, Sophien Kamoun ${ }^{1}$, Vivianne G. A. A. Vleeshouwers $^{2}$ and Jonathan D. G. Jones ${ }^{1 *}$

${ }^{1}$ The Sainsbury Laboratory, University of East Anglia, Norwich Research Park, Norwich, NR4 7UH, UK

${ }^{2}$ Wageningen UR Plant Breeding, Wageningen University and Research, Droevendaalsesteeg 1, 6708 PB, Wageningen, The Netherlands

${ }^{\#}$ Current addresses:

HSK: U.S. Department of Agriculture-Agricultural Research Service, Madison, WI 53706, U.S.A

TS: Institute of Plant Protection, Jiangsu Academy of Agricultural Sciences, Nanjing, 210014, P. R. China

CHW: Institute of Plant and Microbial Biology, Academia Sinica, Taiwan

HA: Nara Institute of Science and Technology, Ikoma 630-0192, Japan

*Corresponding author: Jonathan D. G. Jones (jonathan.jones@tsl.ac.uk) 


\begin{abstract}
Diverse pathogens from the genus Phytophthora cause disease and reduce yields in many crop plants. Although many Resistance to Phytophthora infestans (Rpi) genes effective against potato late blight have been cloned, few have been cloned against other Phytophthora species. Most Rpi genes encode nucleotide-binding domain, leucine-rich repeat- containing (NLR) proteins, that recognize RXLR effectors. However, whether NLR proteins can recognize RXLR effectors from multiple different Phytophthora pathogens has rarely been investigated. Here, we report the effector AVRamr3 from P. infestans that is recognized by Rpi-amr3 from $S$. americanum. We show here that AVRamr3 is broadly conserved in many different Phytophthora species, and that recognition of AVRamr3 homologs enables resistance against multiple Phytophthora pathogens, including P. parasitica and P. palmivora. Our findings suggest a novel path to identifying $R$ genes against important plant pathogens.
\end{abstract}




\section{Introduction}

3 Species in the oomycete genus Phytophthora cause many devastating plant diseases. For example, $P$. infestans, P. parasitica, P. cactorum, P. ramorum, $P$. sojae, P. palmivora and $P$. megakarya cause disease on potato and tomato, tobacco, strawberry, oak, soybean and cacao, respectively (Kamoun et al., 2015).

Plant immunity involves detection of pathogen-derived molecules by either cell-surface pattern recognition immune receptors (PRRs) or intracellular nucleotide-binding domain, leucine-rich repeat containing (NLR) immune receptors, that activate either pattern-triggered immunity (PTI) or effector-triggered immunity (ETI), respectively (Jones and Dangl, 2006). So far, many Resistance to P. infestans (Rpi) genes were cloned from wild Solanum species which confer resistance against potato late blight (Vleeshouwers et al., 2011). Many $R$ genes against $P$. sojae (Rps) have also been mapped in different soybean accessions, and some were cloned (Sahoo et al., 2017). In tobacco, the black shank resistance genes $P h l, P h p$ and $P h$ were genetically mapped but not yet cloned; these confer race-specific resistance to $P$. parasitica (aka $P$. nicotianae) isolates (Gallup and Shew, 2010; Bao et al., 2019). For P. palmivora, some resistant cacao (Theobroma cacao) accessions were identified, but no dominant $R$ genes have been defined or cloned (Thevenin et al., 2012). In summary, very few $R$ genes against other Phytophthora pathogens have been cloned.

Solanum americanum and $S$. nigrum are highly resistant to $P$. infestans (Witek et al., 2016; Witek et al., 2021). Two Rpi genes of coiled-coil (CC) type, Rpi-amr3 and Rpi-amr1, were cloned from different $S$. americanum accessions, and both confer broad-spectrum late blight resistance in cultivated potatoes (Witek et al., 2016; Witek et al., 2021).

In oomycetes, Rpi proteins typically recognize RXLR (Arg-X-Leu-Arg, X represents any amino acid)-EER (Glu-Glu-Arg) effectors that are secreted into plant cells (Rehmany et al., 2005; Wang et al., 2019). Many Avirulence (Avr) genes encoding recognized effectors from Phytophthora species have been identified and they are often fast-evolving and lineage-specific molecules (Jiang et al., 2008). Recently, AVRamr1 (PITG_07569), the recognized effector of Rpi-amr1 was identified by a long read and cDNA pathogen enrichment sequencing (PenSeq) approach (Lin et al., 2020a). Surprisingly, AVRamr1 homologs were identified from $P$. 
34 parasitica and $P$. cactorum genomes and both are recognized by all Rpi-amr1 variants (Witek et al., 2021). Similarly, AVR3a-like effectors were found in different Phytophthora species, including $P$. capsici and $P$. sojae, and the recognition of AVR3a homologs correlates with $P$. capsici or P. sojae resistance in Nicotiana species and soybean (Shan et al., 2004; VegaArreguín et al., 2014), also AVRblb2 homologs from P. andina and P. mirabilis trigger $\mathrm{HR}$ with Rpi-blb (Oliva et al., 2015). Remarkably, a single N336Y mutation in R3a expands its recognition specificity to $P$. capsici AVR3a homolog (Segretin et al., 2014). These reports raise

41 intriguing questions. Could RXLR effectors be widely conserved molecules among different

42 Phytophthora species? Could these effectors be recognized by the same plant receptor? Of 43 particular interest, could this effector recognition capacity enable disease resistance?

45 Here we identified AVRamr3, a novel AVR protein from P. infestans, by screening an RXLR 46 effector library. AVRamr3 is a broadly conserved effector found in many different 47 Phytophthora species. Strikingly, the recognition of AVRamr3 not only enables resistance to $48 \quad P$. infestans, but also to other economically important Phytophthora pathogens including $P$. parasitica and P. palmivora. We also show functional Rpi-amr3 genes are widely distributed among $S$. americanum and S. nigrum accessions, together with the previously defined Rpiamrl genes, they might underpin the "non-host" resistance of these species against $P$. infestans. 


\section{Results:}

\section{Avramr3 encodes a conserved RXLR-WY effector protein}

To identify the effector recognized by Rpi-amr3, we screened an RXLR effector library

(Rietman, 2011; Lin et al., 2020) by Agrobacterium tumefaciens-mediated co-expression with

Rpi-amr3 in Nicotiana benthamiana. By screening 150 RXLR effectors, we found PITG_21190 specifically induces hypersensitive response (HR) with Rpi-amr3 (Fig. 1a), and concluded PITG_21190 is Avramr3. Avramr3 encodes a 339-aa protein with a signal peptide followed by RXLR, EER motifs and four predicted WY motifs (Win et al., 2012) (Fig. 1b). In P. infestans T30-4, the expression of Avramr3 is low during infection, however Avramr3 upregulated in 3928A and US23 in 2-3 days after infection (Cooke et al., 2012; Lin et al., 2020a).

67

Many RXLR effectors are fast-evolving, multiple-member family proteins with extensive sequence polymorphism, such as the Avr2 and Avrblb2 families (Gilroy et al., 2011; Oliva et al., 2015). To study the sequence polymorphism of Avramr3, seventeen additional Avramr 3 homologs from eleven isolates were identified from published databases (KR_1, 3928A, EC1, 6_A1 and US23) (Lee et al., 2020; Lin et al., 2020a) or cloned by PCR (EC1, Katshaar, Pi14538, Pi88069, Pi99183 and Pi99177) (Fig. S1). The sequence alignment shows Avramr3 is a highly conserved RXLR effector among $P$. infestans isolates (Fig. S1).

To find the domain responsible for recognition by Rpi-amr3, ten truncated Avramr3 fragments were cloned (T1 to T10, Fig. 1b, Fig S2) in an expression vector, and transiently co-expressed with Rpi-amr3 in N. benthamiana. We found four AVRamr3 truncations (T3, T4, T8 and T10) can be recognized by Rpi-amr3. T10 (111-240 aa) which carries the $2^{\text {nd }}$ and $3^{\text {rd }}$ WY motifs is the minimal region to be recognized by Rpi-amr3 but not the adjacent T9 protein (130-258 aa) (Fig. 1b and 1c). This suggests these 130 amino-acids of AVRamr3 T10 are sufficient for recognition by Rpi-amr3 and initiation of HR. 

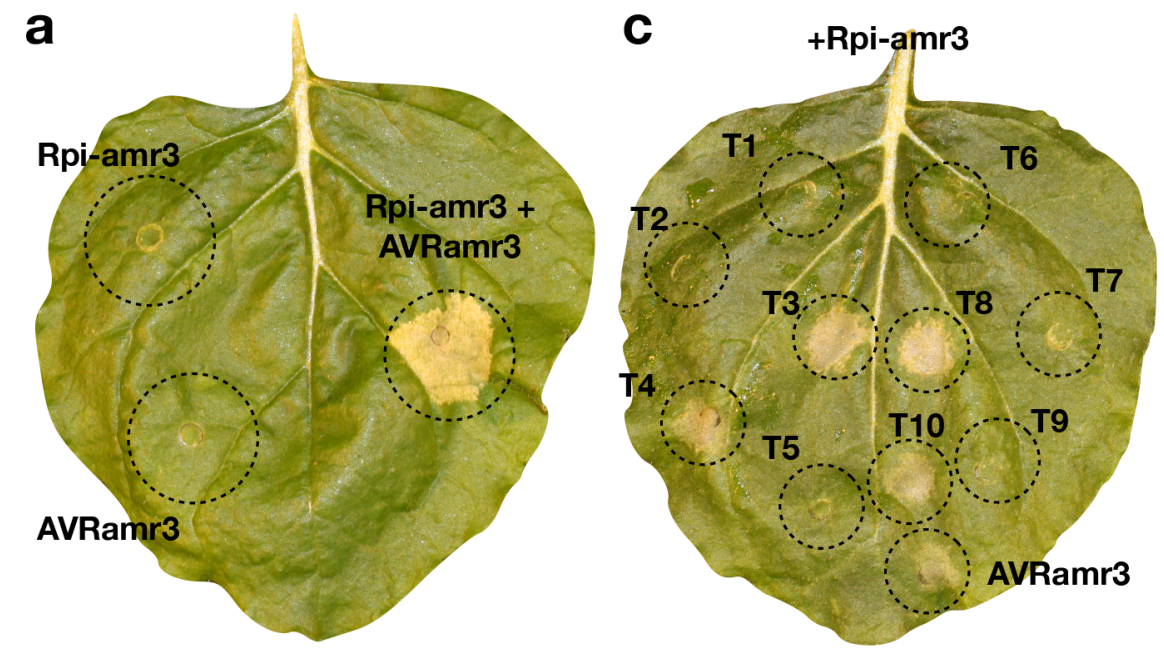

b

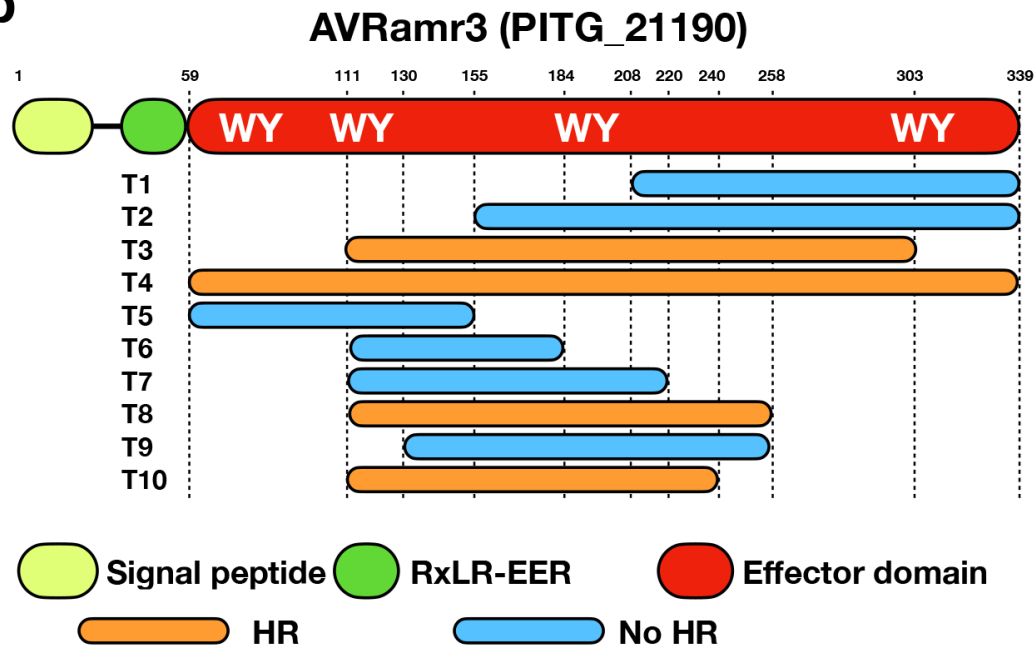

88 Figure 1. AVRamr3 is the recognized effector for Rpi-amr3.

(a). Co-expression of Rpi-amr3::GFP and AVRamr3::HIS-FLAG trigger cell death on $N$. benthamiana, but expression of Rpi-amr3::GFP or AVRarm3::HIS-FLAG individually do not induce cell death. The photo was taken 3 days after infiltration, Agrobacterium strain GV3101(pMP90) carrying Rpi-amr3:GFP or AVRamr3::HISFLAG constructs were used in this experiment. $\mathrm{OD}_{600}=0.5$. Three biological replicates were performed with same results. (b). Cartoon of AVRamr3 (PITG_21190), a protein with 339 amino acids with a signal peptide (lemon), RXLR-EER motif (green), and an effector domain (red) with four predicted WY motifs (Details are shown in Fig. S4). T1-T10 indicate the AVRamr3 truncations used in HR assays. Those that induce HR after co-expression with Rpi-amr3 are marked by orange bars, otherwise by blue. (c). Co-expression of Rpi-amr3::GFP and AVRamr3 truncations, all truncations are tagged with C-terminal HIS-FLAG tag. T3, T4, T8 and T10 trigger cell death when co-expressed with Rpi-amr3, but not T1, T2, T5, T6, T7 and T9. Full-length AVRamr3::HIS-FLAG was used as control. $\mathrm{OD}_{600}=0.5$. Three biological replicates were performed with same results. 
Rpi-amr3 is dependent on the helper NLRs NRC2, NRC3 and NRC4

In Solanaceae, the functionality of many CC-NLR proteins requires helper NLR proteins of the amr3 and Avramr3 in NRC knockout N. benthamiana lines (nrc2/3_1.3.1, nrc4_185.9.1.3, nrc2/3/4_210.4.3)(Adachi et al., 2019; Wu et al., 2020; Witek et al., 2021). As with wild type $N$. benthamiana, we found HR on the nrc2/3_1.3.1 and nrc4_185.9.1.3 knockout lines, but not the nrc2/3/4_210.4.3 knockout line. Similarly, only nrc2/3/4_210.4.3 knockout lines show susceptibility to P. infestans after Rpi-amr3 transient expression (Fig. S3). Therefore, these data suggest both Rpi-amr3-mediated effector recognition and resistance require NRC2, NRC3 or NRC4.

\section{Rpi-amr3 associates with AVRamr3 in planta}

To date, most Rpi proteins recognize their cognate effectors in an indirect manner, except RB and IPI-O effectors (Chen et al., 2012; Kourelis and van der Hoorn, 2018). To test the interaction between Rpi-amr3 and AVRamr3, Rpi-amr3::HA and AVRamr3::HIS-FLAG epitope-tagged constructs were generated and transiently co-expressed in $\operatorname{nrc} 2 / 3 / 4$ knockout $N$. benthamiana leaves to avoid cell death. Protein was then extracted and bi-directional coimmunoprecipitation (Co-IP) was performed. These co-IPs indicate that Rpi-amr3 associates with AVRamr3 bidirectionally (Fig. 2a). We also tested their interaction using a split-luciferase assay. Rpi-amr3::Cluc and AVRamr3::Nluc constructs were transiently expressed in the $\operatorname{nrc} 2 / 3 / 4$ knockout $N$. benthamiana. Luciferase signal was only detected when Rpi-amr3::Cluc and AVRamr3::Nluc were co-expressed. It suggests Rpi-amr3 physically associates with AVRamr3 in-planta (Fig. 2b), but not in negative controls. Our data therefore are consistent with direct interaction of Rpi-amr3 and AVRamr3 proteins, though do not exclude the possible involvement of additional proteins. 

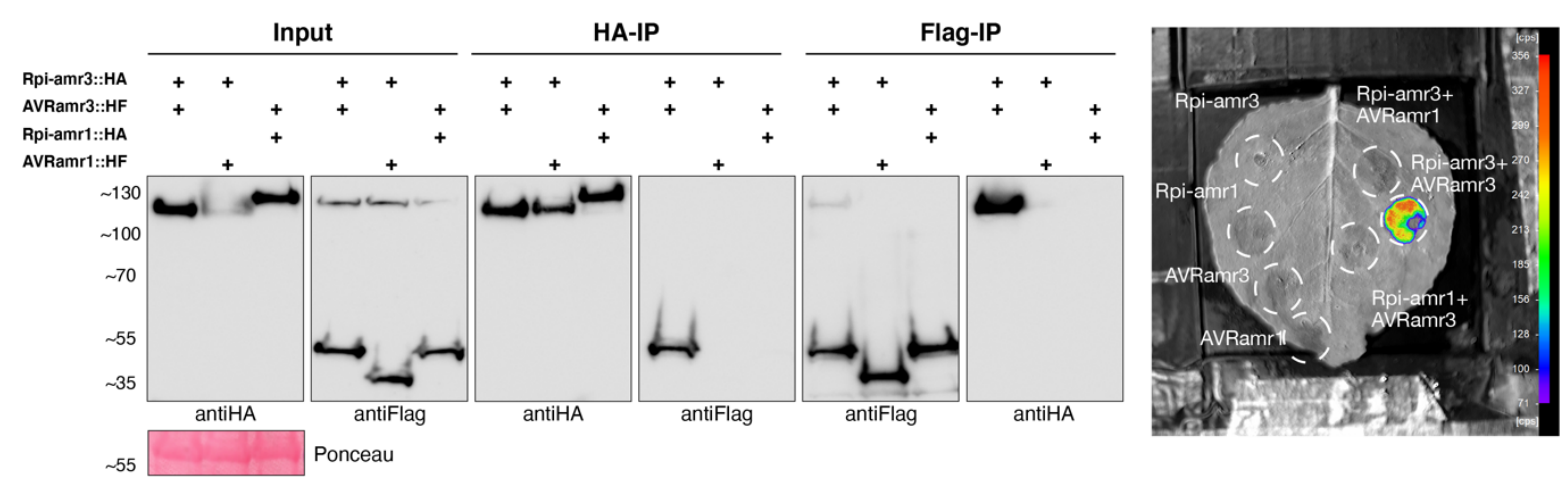

Figure 2. Rpi-amr3 directly interacts with AVRamr3.

140 (a). Rpi-amr3::HA and AVRamr3::HIS-FLAG constructs were used for bidirectional co-immunoprecitation 141 experiment, with Rpi-amr1-HA and AVRamr1::HIS-FLAG used as control. After HA pull down of Rpi-amr3::HA or Rpi-amr1::HA, only AVRamr3::HIS-FLAG is associated with Rpi-amr3::HA. After Flag pull down of AVRamr3::HIS-FLAG or AVRamr1-HIS-FLAG, only Rpi-amr3::HA is associated with AVRamr3::HIS-FLAG. Agrobacterium strain GV3101(pMP90) carrying different constructs were used for transiently expression in nrc2/3/4 knockout Nicotiana benthamiana line (210.4.3) to abolish the cell death phenotype. $\mathrm{OD}_{600}=0.5$. Three biological replicates were performed with same results. (b). Rpi-amr3::Cluc and AVRamr3::Nluc constructs were used to test their interaction in planta, Rpi-amr1::Cluc and AVRamr1::Nluc were used as controls. The luciferase signal can only be detected upon Rpi-amr3::Cluc and AVRamr3::Nluc co-expression.

\section{Avramr3 orthologs occur in multiple Phytophthora species}

To study the evolution of Avramr3 in Phytophthora species, we searched for Avramr3 homologs from published Phytophthora and Hyaloperonospora arabidopsidis (Hpa) genomes. Surprisingly, we found Avramr3 homologs in many Phytophthora genomes, including $P$. parasitica, P. cactorum, $P$. palmivora, $P$. pluvialis, $P$. megakarya, $P$. lichii, $P$. ramorum, $P$.

156 lateralis, P. sojae. P. capsici, P. cinnamomi, and in H. arabidopsidis. Most of the Avramr3 157 homologs are located at a syntenic locus (Fig. 3a). Notably, the P. infestans Avramr3containing contig was not fully assembled; sequences are missing on the 5' side of Avramr3 (Fig. 3a). The protein alignment of the thirteen AVRamr3 homologs is shown in Fig S4. 
a

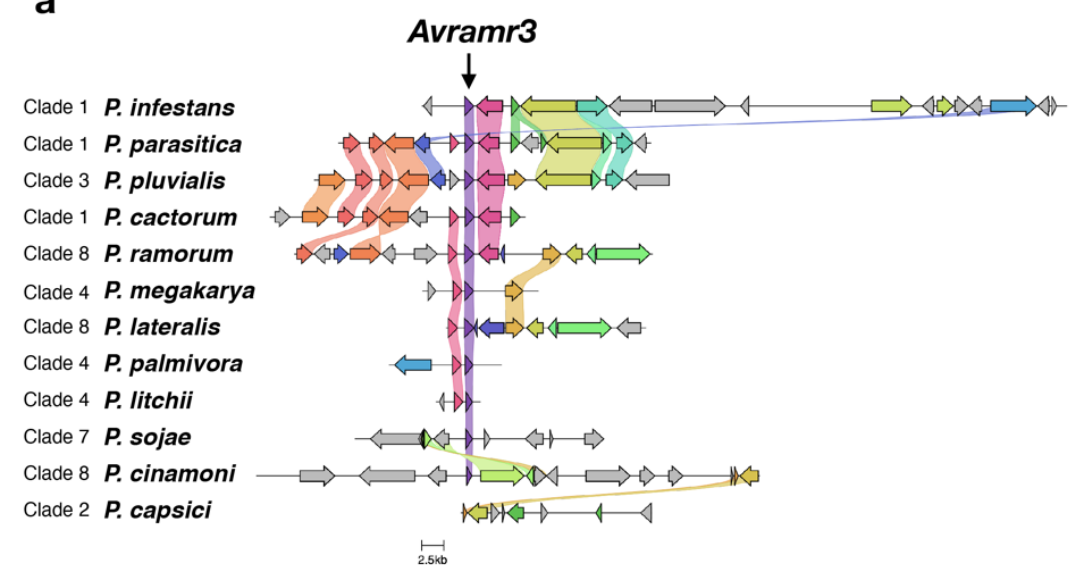

b
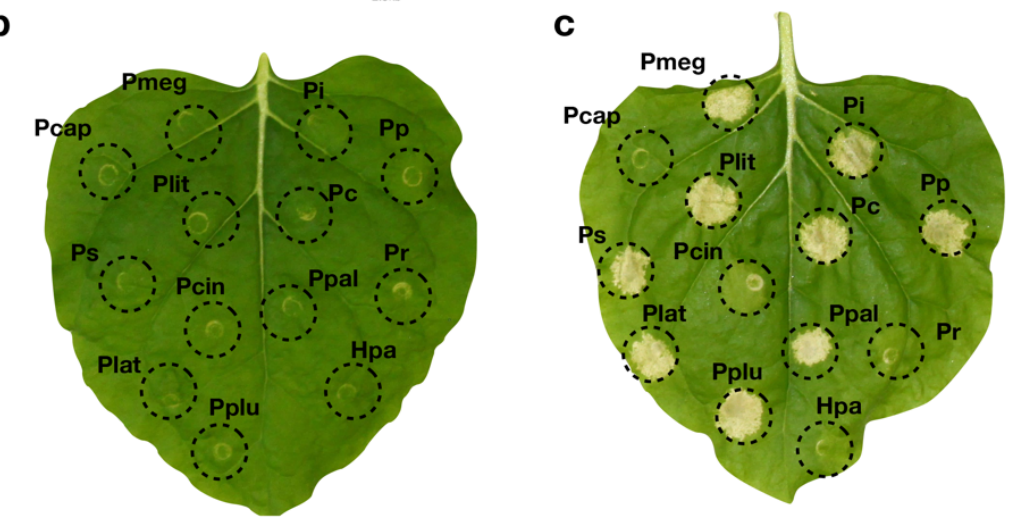

Figure 3. AVRamr3 is a conserved effector among different Phytophthora species.

163 (a). The synteny map of Avramr3 loci from twelve different Phytophthora genomes. The Avramr3 loci were extracted from different genomes, annotated by the gene prediction tool in EumicrobeDB, then analyzed and visualized by Clinker. Avramr3 homologs are shown by purple triangles and indicated by a black arrow, the flanking genes with homology are represented by the corresponding colours. The Phytophthora clades are adapted from Phytophthora database (Rahman et al., 2014). (b). Expression of AVRamr3 homologs with HIS-FLAG tag alone does not trigger cell death on Nicotiana benthamiana. Agrobacterium strain GV3101(pMP90) carring different constructs were used in this experiment. $\mathrm{OD}_{600}=0.5$. Three biological replicates were performed with same results.

171 (c). Co-expression of AVRamr3 homologs with Rpi-amr3::GFP in N. benthamiana. The AVRamr3 homologs 172 from Phytophthora infestans $(\mathrm{Pi})$, P. parasitica $(\mathrm{Pp})$, P.cactorum $(\mathrm{Pc})$, P. palmivora $(\mathrm{Ppal})$, P. megakarya $(\mathrm{Pmeg})$,

173 P. litchi (Plit), P. sojae (Ps), P. lateralis (Plat) and P. pluvialis (Pplu) induce cell death after co-expression with

174 Rpi-amr3::GFP, but not AVRamr3 homologs from P. ramorum (Pr), P. capsici (Pcap) and Hyaloperonospora arabidopsidis (Hpa). The AVRamr3 homolog from P. cinnamomi (Pcin) shows an intermediate cell death. Agrobacterium strain GV3101(pMP90) carrying different constructs were used in this experiment. $\mathrm{OD}_{600}=0.5$. Three biological replicates were performed with same results.

179 To test if those AVRamr3 homologs from different Phytophthora species are also recognized 180 by Rpi-amr3, we synthesized and cloned them into an expression vector with the $35 \mathrm{~S}$ promoter, 181 and performed transient expression assays in N. benthamiana. Expressing the effectors alone 
does not trigger HR in $N$. benthamiana (Fig. 3b), but AVRamr3 homologs from P. parasitica, P. cactorum, P. palmivora, P. megakarya, P. lichii, P. sojae, P. lateralis and P. pluvialis can induce HR when co-expressed with Rpi-amr3. The AVRamr3 homolog from P. cinnamomi triggers an intermediate HR, and the AVRamr3 homologs from P. ramorum, P. capsici, and $H$. arabidopsidis (Fig 3c) do not trigger Rpi-amr3-dependent HR.

To test if particularly conserved amino-acids of AVRamr3 are responsible for the Rpi-amr3 recognition, we mutated eight conserved amino-acid on the AVRamr3 T10 region (Figure S4). However, all tested mutants are still recognized by Rpi-amr3 (Figure S5). This result indicates the recognition specificity might not be determined by any single amino acid on AVRamr3, but rather by its overall structure.

To test if other recognized AVRamr3 homologs also directly interact with Rpi-amr3, we performed co-immunoprecipitation and split-luciferase assays in nrc2/3/4_210.4.3 knockout lines. We found all the recognized AVRamr3 homologs associate with Rpi-amr3 by coimmunoprecipitation, though with varied affinity. Two unrecognized AVRamr3 homologs from $P$. capsici and $H$. arabidopsidis do not associate with Rpi-amr3. However, two unrecognized or weakly recognized AVRamr3 homologs from P. ramorum and P. cinnamomi also associate with Rpi-amr3, and the unrecognized AVRamr3-T9 truncation shows a weak association (Figure S6). In contrast, the output of split-luciferase assay is fully consistent with the HR assay (Figure S7). Our data indicating that an in-planta receptor-ligand interaction is necessary but might not be sufficient for the activation of Rpi-amr3 and triggering of HR.

Rpi-amr3 confers resistance to multiple $P$. parasitica and $P$. palmivora strains in $N$. benthamiana

Rpi-amr3 was previously reported to confer resistance against potato late blight caused by $P$. infestans (Witek et al., 2016). Its broad effector recognition capacity suggested Rpi-amr3 might confer resistance against additional Phytophthora pathogens.

212 To test this hypothesis, we generated Rpi-amr3 stable transformed $N$. benthamiana lines. Two

213 homozygous T2 lines \#13.3 and \#16.5 were verified to confer $P$. infestans resistance and 214 evaluated for P. parasitica and P. palmivora resistance. Both these pathogens have a wide host 215 range, including the model plant $N$. benthamiana. 
217 Six P. parasitica isolates (R0, R1, 310, 666, 329 and 721) were tested on N. benthamiana

218 carrying Rpi-amr3, and on wild type N. benthamiana plants as negative control. A suspension

219 of zoospores was used for root inoculation (Material and Methods). We found both $N$.

220 benthamiana - Rpi-amr3 lines resist three $P$. parasitica isolates R1, 666 and 721, but are

221 susceptible to R0, 310 and 329 (Fig 4). In summary, Rpi-amr3 confers resistance against three

222 out of six tested $P$. parasitica isolates in N. benthamiana.

WT
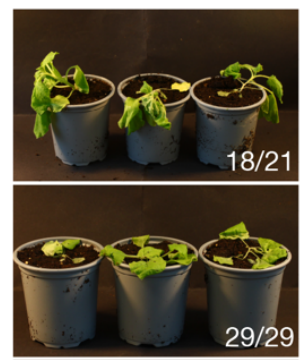

$29 / 29$
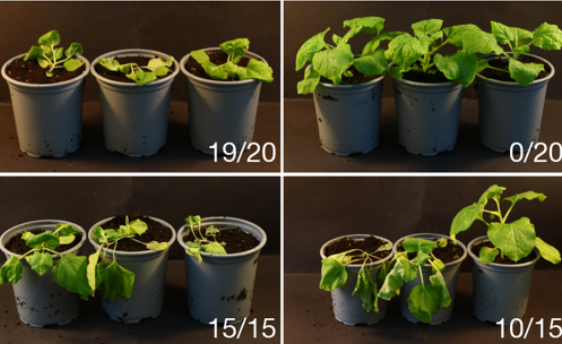

$0 / 20$
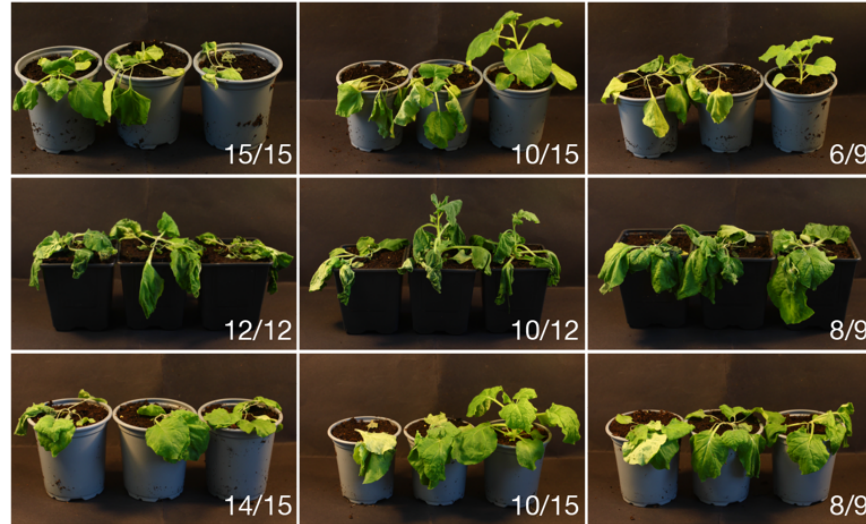

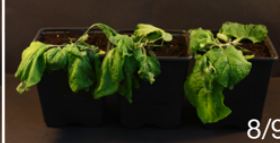

Rpi-amr3\#16.5

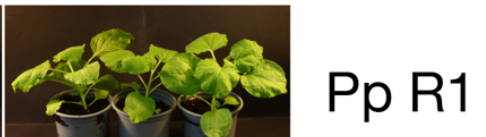

Pp 666

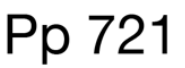

Pp R0
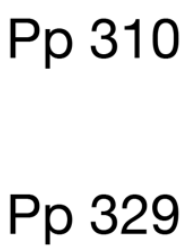

Figure 4. Root inoculation of six Phytophthora parasitica isolates on Rpi-amr3 transgenic

\section{Nicotiana benthamiana lines.}

Representative photos for the $P$. parasitica root inoculation tests are shown. Two homozygous $N$. benthamiana Rpi-amr3 lines \#13.3 and \#16.5 were used in this experiment. Wild type $N$. benthamiana plants were used as control. Six P. parasitica isolates were used for root inoculation, Rpi-amr 3 confers resistance against R1, 666 and 721, but not R0, 310 and 329. 3-4 weeks $N$. benthamiana were used for the root inoculation, 3 plants/line were used for each experiment and as least three biological replicates were performed with similar results The numbers indicate susceptible plants/total tested plants.

233 The PpAvramr3 homologs from the six P. parasitica isolates were PCR amplified, sub-cloned 234 and sequenced. PpAvramr3 homologs were identified from R0, R1 and 310, 666 and 721, but 
not from 329 (Fig S8). These data suggest the presence of recognized AVRamr3 homologs

236 from the Phytophthora pathogens is necessary but not sufficient to induce Rpi-amr3 mediated

237 resistance.

238

239 Furthermore, we tested another broad host range Phytophthora pathogen, P. palmivora, which

240 causes major losses on many tropical tree crops like papaya, mango, cacao, coconut and palm

241 tree. We tested seven P. palmivora isolates on the two Rpi-amr3 transgenic $N$. benthamiana

242 lines by root inoculation, and wild type $N$. benthamiana was used as a control. We found Rpi-

$243 a m r 3$ confers resistance to three out of seven tested P. palmivora isolates, including 7551, 7547,

2447545 , but not to 3914, 7548. For two other isolates 0113 and 3738, inconsistent results were

245 obtained from the two Rpi-amr3 transgenic lines (Fig 5). To verify the presence of Avramr3

246 homologs in these tested P. palmivora isolates, we PCR amplified the Avramr3 homologs from

247 genomic DNA of the seven $P$. palmivora isolates. All the tested $P$. palmivora carry

248 PpalAvramr3 variants (Figure S9). Taken together, Rpi-amr3 confers resistance to at least 3/7

249 tested $P$. palmivora isolates in the root inoculation assay.

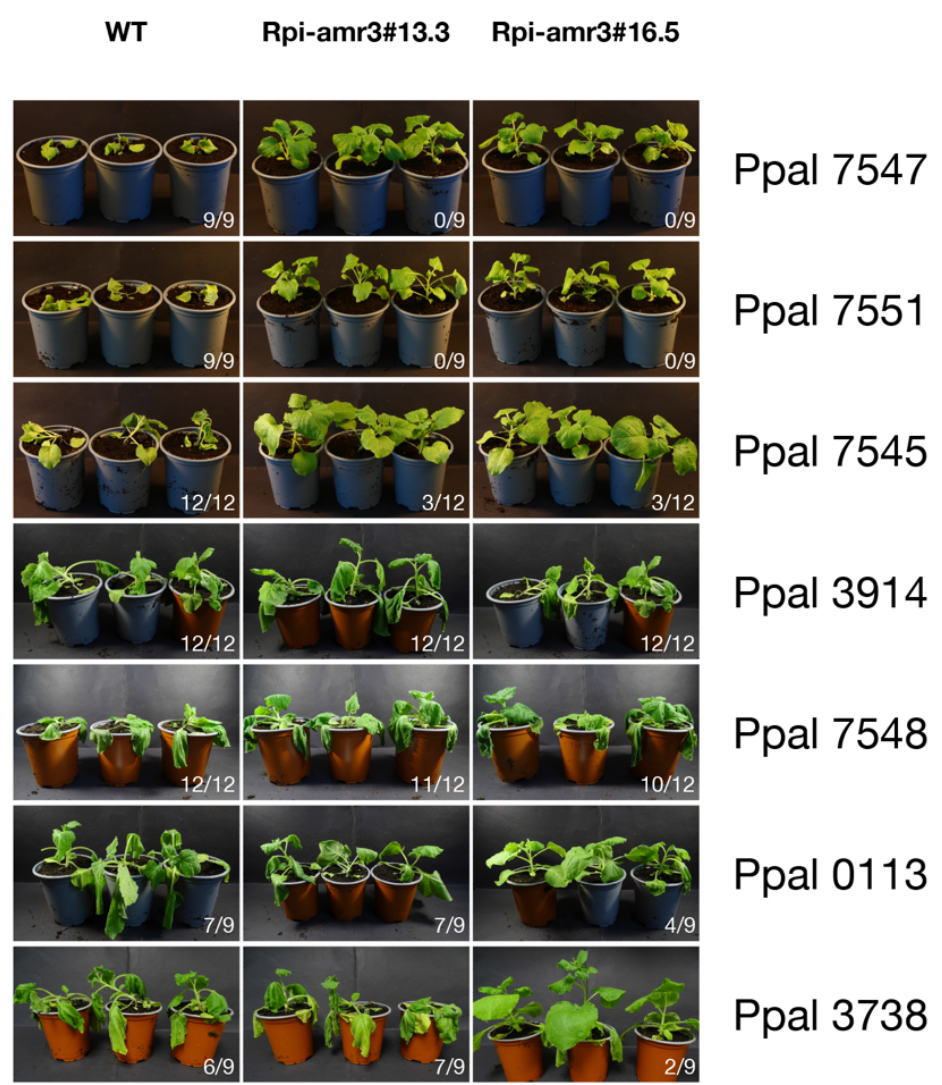

251 Figure 5. Root inoculation of 7 Phytophthora palmivora isolates on Rpi-amr3 transgenic

$252 \quad$ Nicotiana benthamiana lines. 
Two homozygous $N$. benthamiana - Rpi-amr3 lines \#13.3 and \#16.5 were used in this experiment, wild type $N$. benthamiana were used as control. Seven P. parasitica isolates were used for root inoculation, Rpi-amr3 confer resistance against isolates 7547,7551 and 7545 , but not 3914, 7548. For isolates 0113 and 3738, we obtained some variable results for the two transgenic lines. 3-4 old weeks $N$. benthamiana were used for the root inoculation, 3 plants/line were used for each experiment and three or more biological replicates were performed with similar results.

Rpi-amr3 is widely distributed in $S$. americanum and $S$. nigrum

Though susceptible accessions can be identified in detached leaf assays, most $S$. americanum and $S$. nigrum accessions show complete resistance in the field to P. infestans. Previously, many functional Rpi-amrl alleles were cloned from different $S$. americanum and S. nigrum accessions (Witek et al., 2021).

The identification of AVRamr3 allows us to investigate the distribution of Rpi-amr3 from all

S. americanum and S. nigrum accessions. In total, 54 S. americanum accessions and $26 S$. amr3. We found 43/54 tested S. americanum accessions show HR after AVRamr3 agroinfiltration (Fig 6a). Similarly, 21/26 tested S. nigrum accessions recognize AVRamr3 (Fig 6b).

To further investigate the sequence polymorphism of Rpi-amr3 from different accessions, the Rpi-amr3 homologs from 14 accessions were extracted from PacBio RenSeq dataset (Witek et al., 2021), including eleven accessions (SP1123, SP2272, SP2273, SP2307, SP2360, SP3399, SP3400, SP1101, SP3406 and SP3409) which respond to AVRamr3 and three accessions (SP1032, SP2271 and SP2275) that do not respond to AVRamr3.

To test the functionality of Rpi-amr3 from S. americanum and S. nigrum, Rpi-amr3 homologs were PCR amplified from gDNA of three S. americanum accessions SP2272, SP2273 and SP3406, and from gDNA of two S. nigrum accessions SP1088 and SP1084. Rpi-amr3 alleles (Rpi-nig3 hereafter) were amplified from each of these two $S$. nigrum accessions and cloned into an expression vector with $35 \mathrm{~S}$ promoter. We found all the seven Rpi-amr3/Rpi-nig3 genes can recognize AVRamr3 in transient assays (Fig 6d), but not the negative control AVRamr1. Compared to Rpi-amr3 from SP1102, the amino-acid identity ranges from $82.2 \%$ to $95.7 \%$ (Fig 6c). Premature stop codons were found in Rpi-amr3 homologs from SP2271 and SP2275 (Figure S10), which result in loss of Rpi-amr3 function. 
Taken together, these data suggest Rpi-amr3 gene is widely distributed in diploid $S$.

a

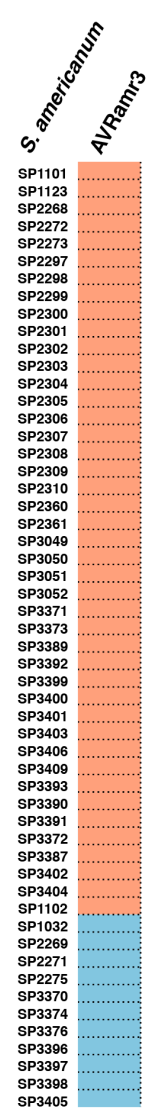

b

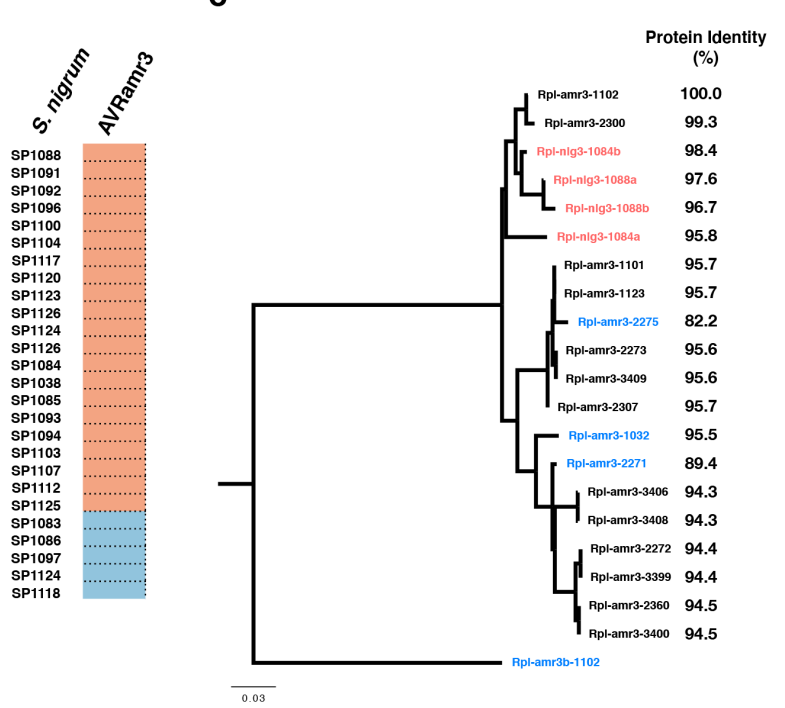

d

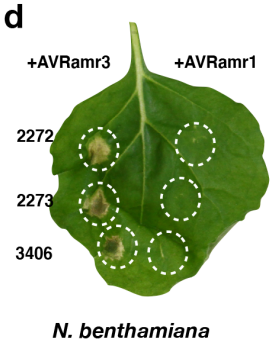

c

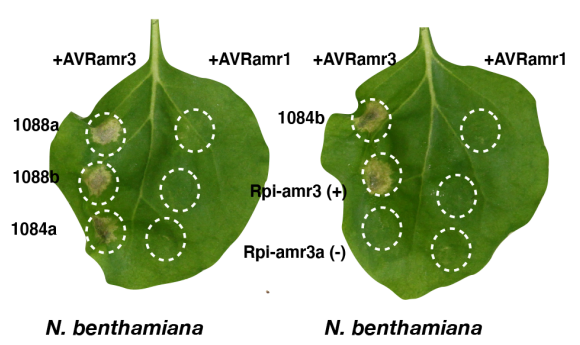

Figure 6. Screen for AVRamr3 recognition on $S$. americanum and $S$. nigrum accessions.

294 (a). 54 S. americanum accessions were screened with Agrobacterium strain GV3101(pMP90) carrying 35S::AVRamr3. The accessions with cell death upon agro-infiltration are marked by red, otherwise blue. (b). 26 S. nigrum accessions were screened with Agrobacterium strain GV3101(pMP90) carrying 35S::AVRamr3. The accessions with cell death upon agro-infiltration are marked by red, otherwise blue. (c). Maximum likelihood (ML) tree of Rpi-amr3 and Rpi-nig3 proteins was made by iqtree with GTT+G4 model. The Rpi-amr3 homologs from S. americanum were extracted from PacBio RenSeq assemblies (Witek et al., 2021). The four Rpi-nig3 genes were PCR amplified from S. nigrum accession SP1088 and SP1084 (red). The non-functional Rpi-amr3 homologs were marked by blue. Rpi-amr3b from SP1102 is a paralogue of Rpi-amr3, which was used as an outgroup of the phylogenetic analysis. The scale bar indicates the number of amino acid substitutions per site. The protein identities of each homolog compared to Rpi-amr3 (Rpi-amr3-1102) are shown by \%. (d). Selected Rpi-amr3 homologs were cloned from three S. americanum accessions SP2272, SP2273, SP3406. Four Rpi-nig3 homologs were cloned from S. nigrum accessions SP1088 and SP1084, and co-expressed with AVRamr3 or AVRamr1 (negative control). All of them can recognize AVRamr3 in the transient assay but not AVRamr1. 


\section{Discussion}

310 In this study, by screening an RXLR effector library of Phytophthora infestans, we identified 311 and characterized a novel effector AVRamr3 (PITG_21190) that is recognized by the NLR

312 protein Rpi-amr3 of $S$. americanum. AVRamr3 is very conserved among all tested $P$. infestans 313 isolates, and AVRamr3 homologs were identified in twelve additional Phytophthora and 314 Hyaloperonospora arabidopsidis genomes. These homologs are located in a syntenic region 315 (Fig. 3a). Surprisingly, we found 9/13 tested AVRamr3 homologs can be recognized by Rpiamr3 leading to HR in N. benthamiana. This finding suggests AVRamr3 is an essential effector among Phytophthora species, though its virulence function has yet to be determined.

319 According to the "zigzagzig" model of plant immunity (Jones and Dangl, 2006), the surface immune receptors like receptor-like proteins (RLPs) and receptor-like kinases (RLKs) perceive relatively conserved microbe-associated molecular patterns (MAMPs) and induce patterntriggered immunity (PTI). Intracellular nucleotide-binding and leucine-rich repeat immune receptors (NLRs) recognize fast-evolving and lineage-specific effectors and activate effectortriggered immunity (ETI). Therefore, PTI was believed to confer broader-spectrum resistance compared to ETI. Indeed, many RLPs/RLKs recognize conserved ligands and /or confer broadspectrum resistance, such as FLS2, EFR, RLP23, RXEG1 and ELR (Zipfel et al., 2006; Albert et al., 2015; Du et al., 2015; Wang et al., 2018). Remarkably, EFR from Arabidopsis thaliana enhances resistance against a range of bacterial pathogens in different crop plants, like tomato, orange and apple (Lacombe et al., 2010; Mitre et al., 2021; Piazza et al., 2021). However, apoplastic effectors can also be fast-evolving proteins, like the SCR74 family in P. infestans (Liu et al., 2005; Lin et al., 2020b), or Cladosporium fulvum AVR2, AVR4 and AVR9 (Joosten et al., 1994; Van den Ackerveken et al., 1994; Luderer et al., 2002; Westerink et al., 2004). On the other hand, MAMP-like cytoplasmic effectors/effector epitopes have been reported. For example, $\mathrm{Sw}-5 \mathrm{~b}$ from tomato confers broad-spectrum tospovirus resistance by recognizing a conserved, 21-amino acid epitope $\mathrm{NSm}^{21}$ which derives from the viral movement protein NSm (Zhu et al., 2017). A recent functional pan-genome study revealed the ETI landscape of $A$. thaliana and Pseudomonas syringae; some P. syringae effectors are widely conserved.

338 Similarly, the ETI mediated by two conserved NLRs CAR1 and ZAR1 confers resistance to 94.7\% P. syringae strains (Laflamme et al., 2020). These observations, as well as our finding 
341 can also be relatively invariant and conserved, and might contribute to broad-spectrum pathogen resistance.

344 To test this hypothesis, we established a $N$. benthamiana root inoculation system by using stable Rpi-amr3 transgenic plants, and tested $P$. parasitica and $P$. palmivora which have a broad host range, and cause dramatic yield losses of many crops from different families (Meng et al., 2014; Ali et al., 2017). Importantly, we found Rpi-amr3 does confer resistance against some $P$. parasitica and $P$. palmivora isolates. This is the first report of cloned $R$ genes against P. parasitica and P. palmivora (Kourelis et al., 2021). Additionally, it is noteworthy that, in nature, many Phytophthora pathogens can co-inoculate the host and interspecific hybridization might occur; for example, $P$. andina was proposed to have emerged through hybridization of $P$. infestans and an unknown Phytophthora species (Goss et al., 2011). Natural hybrids of $P$. parasitica and $P$. cactorum were also found on infected loquat trees (Hurtado-Gonzales et al., 2017). An $\mathrm{R}$ protein that provides protection against both foliar and root Phytophthora pathogens of different species would be extremely valuable. However, some Rpi-amr3 breaking P. parasitica and P. palmivora strains were also identified in our study, although most of them carry the recognized AVRamr3 homologs. This might be caused by silencing of the recognized effector gene like Avrvnt 1 to avoid the recognition by Rpi-vnt1, or presence of other suppressors or regulators like Avrcap1b or splicing regulatory (SRE) effectors (Pais et al., 2018; Huang et al., 2020; Derevnina et al., 2021).

In this study, we also reported that Rpi-amr3 directly interacts with AVRamr3 and other recognized AVRamr3 homologs from different Phytophthora species. Surprisingly, the direct interaction has not led to accelerated evolution of Avramr3 to evade detection, as we also observed for Rpi-amr1 and AVRamr1 (Lin et al., 2020a; Witek et al., 2021). This could predispose Rpi-amr3 to function in different plant species.

368 Thus, Rpi-amr3 could be deployed in Solanaceae crops like potato, tomato and tobacco against multiple Phytophthora diseases. However, interfamily transfer of NLR genes remains a challenge if $N L R$ genes show "restricted taxonomic functionality" (Tai et al., 1999). The paired NLR genes RPS4/RRS1 from Brassicaceae (Arabidopsis) can nevertheless function in other plant families like Solanaceae (tomato) and Cucurbitaceae (cucumber) against different bacterial and fungal diseases (Narusaka et al., 2013). Here, we found any of the NRC2, NRC3 or NRC4 proteins are required for Rpi-amr3 to execute its function. Thus, in the plant families 
375

376

377

378

379

380

381

382

383

384

385

386

387

388

389

390

391

392

393

394

395

396

397

398

399

400

401

402

403

404

405

which lacking NRC homologs, such as tropical tree crops susceptible to $P$. palmivora and $P$. megakarya, co-delivery of Rpi-amr3 and NRC genes might be required to defeat these Phytophthora diseases.

"Non-host" resistance is durable. S. americanum and S. nigrum are thought to be non-host plants of $P$. infestans, although susceptible accessions of both species have been found using DLAs. This opens the opportunity to dissect their "non-host" resistance. By using AVRamr3 as a probe, we found Rpi-amr3 is widely distributed in $S$. americanum and $S$. nigrum species (Witek et al., 2021) (Fig. 6). We noticed that PITG_21190 (AVRamr3) triggers HR in many $S$. nigrum accessions in a large-scale effector screening study (Dong, 2016), consistent with our findings (Fig. 6). Furthermore, we cloned four Rpi-nig3 genes from two $S$. nigrum accessions. All the four Rpi-nig3 homologs recognize AVRamr3 in our co-expression assays (Fig. 6), although their resistance to late blight needs to be evaluated individually. The wide distribution of Rpi-amr3 and Rpi-amrl suggests that these two $R$ genes, perhaps with other $R$ genes and the NRC network in S. americanum and S. nigrum, underpin their "non-host" resistance against potato late blight. The identification of AVRamr3 and AVRamr1 can also help to explore other novel resistance genes from S. americanum and S. nigrum.

In summary, this study reveals that $R p i-a m r 3$ is a conserved and broad-spectrum $R$ gene from S. americanum and its relatives. The recognition of the conserved AVRamr3 effectors leads to resistance against several different Phytophthora pathogens. This finding shows great potential for resistance breeding in many crop plants against different Phytophthora diseases.

(1)

(1)

(1)




\section{References}

407

408

409

410

411

412

413

414

415

416

417

418

419

420

421

422

423

424

425

426

427

428

429

430

431

432

433

434

435

436

437

438

439

440

441

442

443

444

Adachi H, Contreras MP, Harant A, Wu C-H, Derevnina L, Sakai T, Duggan C, Moratto E, Bozkur TO, Maqbool A, et al (2019) An N-terminal motif in NLR immune receptors is functionally conserved across distantly related plant species. eLife 8: 121

Albert I, Böhm H, Albert M, Feiler CE, Imkampe J, Wallmeroth N, Brancato C, Raaymakers TM, Oome S, Zhang H, et al (2015) An RLP23-SOBIR1-BAK1 complex mediates NLP-triggered immunity. NPLANTS 1: 15140

Ali SS, Shao J, Lary DJ, Strem MD, Meinhardt LW, Bailey BA (2017) Phytophthora megakarya and P. palmivora, causal agents of black pod rot, induce similar plant defense responses late during infection of susceptible Cacao pods. Frontiers in Plant Science 8: 36

Bao Y, Ding N, Qin Q, Wu X, Martinez N, Miller R, Zaitlin D, Li D, Yang S (2019) Genetic mapping of the $P h$ gene conferring disease resistance to black shank in tobacco. Mol Breeding 1-10

Cooke DEL, Cano LM, Raffaele S, Bain RA, Cooke LR, Etherington GJ, Deahl KL, Farrer RA, Gilroy EM, Goss EM, et al (2012) Genome analyses of an aggressive and invasive lineage of the Irish potato famine pathogen. PLoS Pathog 8: e1002940

Derevnina L, Contreras MP, Adachi H, Upson J, Cruces AV, Xie R, Sklenar J, Menke FLH, Mugford ST, Maclean D, et al (2021) Plant pathogens convergently evolved to counteract redundant nodes of an NLR immune receptor network. Biorxivorg: 2021.02.03.429184

Dong R (2016) Identification of the function of four genes encoding the RxLR secreted protein in Phytophthora infestans. PhD thesis. Shandong Agricultural University

Du J, Verzaux E, Chaparro-Garcia A, Bijsterbosch G, Keizer LCP, Zhou J, Liebrand TWH, Xie C, Govers F, Robatzek S, et al (2015) Elicitin recognition confers enhanced resistance to Phytophthora infestans in potato. NPLANTS 10.1038-nplants.2015.34

Gallup CA, Shew HD (2010) Occurrence of race 3 of Phytophthora nicotianae in North Carolina, the causal agent of black shank of tobacco. Plant Disease 94: 557-562

Gilroy EM, Breen S, Whisson SC, Squires J, Hein I, Kaczmarek M, Turnbull D, Boevink PC, Lokossou A, Cano LM, et al (2011) Presence/absence, differential expression and sequence polymorphisms between PiAVR2 and PiAVR2-like in Phytophthora infestans determine virulence on $R 2$ plants. New Phytol 191: 763-776

Goss EM, Cardenas ME, Myers K, Forbes GA, Fry WE, Restrepo S, Grünwald NJ (2011) The plant pathogen Phytophthora andina emerged via hybridization of an unknown Phytophthora species and the Irish potato famine pathogen, $P$. infestans. PLoS ONE 6: e24543

Huang J, Lu X, Wu H, Xie Y, Peng Q, Gu L, Wu J, Wang Y, Reddy ASN, Dong S (2020) Phytophthora effectors modulate genome-wide alternative splicing of host mRNAs to reprogram plant immunity. Molecular Plant 13: 1470-1484 
Hurtado-Gonzales OP, Aragon-Caballero LM, Flores-Torres JG, Veld WMIT, Lamour KH (2017) Molecular comparison of natural hybrids of Phytophthora nicotianae and $P$. cactorum infecting loquat trees in Peru and Taiwan. Mycologia 101: 496-502

Jiang RHY, Tripathy S, Govers F, Tyler BM (2008) RXLR effector reservoir in two Phytophthora species is dominated by a single rapidly evolving superfamily with more than 700 members. Proceedings of the National Academy of Sciences 105: 4874-4879

Jones JDG, Dangl JL (2006) The plant immune system. Nature 444: 323-329

Joosten MHAJ, Cozijnsen TJ, de Wit PJGM (1994) Host resistance to a fungal tomato pathogen lost by a single base-pair change in an avirulence gene. Nature 367: 384

Kamoun S, Furzer O, Jones JDG, Judelson HS, Ali GS, Dalio RJD, Roy SG, Schena L, Zambounis A, Panabières F, et al (2015) The Top 10 oomycete pathogens in molecular plant pathology. Molecular Plant Pathology 16: 413-434

Kourelis J, Sakai T, Adachi H, Kamoun S (2021) RefPlantNLR: a comprehensive collection of experimentally validated plant NLRs. Biorxivorg: 2020.07.08.193961

Lacombe S, Rougon-Cardoso A, Sherwood E, Peeters N, Dahlbeck D, van Esse HP, Smoker M, Rallapalli G, Thomma BPHJ, Staskawicz B, et al (2010) Interfamily transfer of a plant pattern-recognition receptor confers broad-spectrum bacterial resistance. Nature Biotechnology 28: 365

Laflamme B, Dillon MM, Martel A, Almeida RND, Desveaux D, Guttman DS (2020) The pan-genome effector-triggered immunity landscape of a host-pathogen interaction. Science 367: $763-768$

Lin X, Song T, Fairhead S, Witek K, Jouet A, Jupe F, Witek AI, Karki HS, Vleeshouwers VGAA, Hein I, et al (2020a) Identification of Avramr1 from Phytophthora infestans using long read and cDNA pathogen-enrichment sequencing (PenSeq). Molecular Plant Pathology 18: 547-11

Lin X, Wang S, de Rond L, Bertolin N, Wouters RHM, Wouters D, Domazakis E, Bitew MK, Win J, Dong S, et al (2020b) Divergent evolution of PcF/SCR74 effectors in oomycetes is associated with distinct recognition patterns in Solanaceous plants. MBio 11: 380

Liu Z, Bos JIB, Armstrong M, Whisson SC, da Cunha L, Torto-Alalibo T, Win J, Avrova AO, Wright F, Birch PRJ, et al (2005) Patterns of diversifying selection in the phytotoxin-like scr74 gene family of Phytophthora infestans. Mol Biol Evol 22: 659-672

Luderer R, Takken FLW, de Wit PJGM, Joosten MHAJ (2002) Cladosporium fulvum overcomes $C f$-2-mediated resistance by producing truncated AVR2 elicitor proteins. Molecular Microbiology 45: 875-884

Meng Y, Zhang Q, Ding W, Shan W (2014) Phytophthora parasitica: a model oomycete plant pathogen. Mycology 5: 43-51

Mitre LK, Teixeira-Silva NS, Rybak K, Magalhães DM, de Souza-Neto RR, Robatzek S, Zipfel C, de Souza AA (2021) The Arabidopsis immune receptor EFR increases resistance 
to the bacterial pathogens Xanthomonas and Xylella in transgenic sweet orange. Plant Biotechnology J. doi: 10.1111/pbi.13629

Narusaka M, Kubo Y, Hatakeyama K, Imamura J, Ezura H, Nanasato Y, Tabei Y, Takano Y, Shirasu K, Narusaka Y (2013) Interfamily transfer of dual NB-LRR genes confers resistance to multiple pathogens. PLoS ONE 8: e55954

Oliva RF, Cano LM, Raffaele S, Win J, Bozkur TO, Belhaj K, Oh S-K, THINES M, Kamoun S (2015) A recent expansion of the RXLR effector gene Avrblb2 is maintained in global populations of Phytophthora infestans indicating different contributions to virulence. MPMI 28: 901-912

Pais M, Yoshida K, Giannakopoulou A, Pel MA, Cano LM, Oliva RF, Witek K, Lindqvist-Kreuze H, Vleeshouwers VGAA, Kamoun S (2018) Gene expression polymorphism underpins evasion of host immunity in an asexual lineage of the Irish potato famine pathogen. BMC Evol Biol 18: 1-11

Piazza S, Campa M, Pompili V, Costa LD, Salvagnin U, Nekrasov V, Zipfel C, Malnoy M (2021) The Arabidopsis pattern recognition receptor EFR enhances fire blight resistance in apple. Biorxivorg: 2021.01.22.427734

Rahman MZ, Uematsu S, Takeuchi T, Shirai K, Ishiguro Y, Suga H, Kageyama K (2014) Two new species, Phytophthora nagaii sp. nov. and P. fragariaefolia sp. nov., causing serious diseases on rose and strawberry plants, respectively, in Japan. J Gen Plant Pathol 80: $348-365$

Rehmany AP, Gordon A, Rose LE, Allen RL, Armstrong MR, Whisson SC, Kamoun S, Tyler BM, Birch PRJ, Beynon JL (2005) Differential recognition of highly divergent downy mildew avirulence gene alleles by RPP1 resistance genes from two Arabidopsis lines. The Plant Cell 17: 1839-1850

Sahoo DK, Abeysekara NS, Cianzio SR, Robertson AE, Bhattacharyya MK (2017) A novel Phytophthora sojae resistance Rps 12 gene mapped to a genomic region that contains several Rps genes. PLoS ONE 12: e0169950

Segretin ME, Pais M, Franceschetti M, Chaparro-Garcia A, Bos JIB, Banfield MJ, Kamoun S (2014) Single amino acid mutations in the potato immune receptor R3a expand response to Phytophthora effectors. MPMI 27: 624-637

Shan W, Cao M, Leung D, Tyler BM (2004) The Avrlb locus of Phytophthora sojae encodes an elicitor and a regulator required for avirulence on soybean plants carrying resistance gene Rps 1b. MPMI 17: 394-403

Tai TH, Dahlbeck D, Clark ET, Gajiwala P, Pasion R, Whalen MC, Stall RE, Staskawicz BJ (1999) Expression of the Bs2 pepper gene confers resistance to bacterial spot disease in tomato. Proceedings of the National Academy of Sciences 96: 14153-14158

Thevenin J-M, Rossi V, Ducamp M, Doare F, Condina V, Lachenaud P (2012) Numerous clones resistant to Phytophthora palmivora in the "Guiana" genetic group of Theobroma cacao L. PLoS ONE 7: e40915 
Van den Ackerveken GFJM, Dunn RM, Cozijnsen AJ, Vossen JPMJ, Van den Broek HWJ, de Wit PJGM (1994) Nitrogen limitation induces expression of the avirulence gene avr9 in the tomato pathogen Cladosporium fulvum. Mol Gen Genet 243: 277-285

Vega-Arreguín JC, Jalloh A, Bos JI, Moffett P (2014) Recognition of an Avr3a homologue plays a major role in mediating nonhost resistance to Phytophthora capsici in Nicotiana species. MPMI 27: 770-780

Vleeshouwers VGAA, Raffaele S, Vossen JH, Champouret N, Oliva R, Segretin ME, Rietman H, Cano LM, Lokossou A, Kessel G, et al (2011) Understanding and exploiting late blight resistance in the age of effectors. Annu Rev Phytopathol 49: 507-531

Wang S, McLellan H, Bukharova T, He Q, Murphy F, Shi J, Sun S, van Weymers P, Ren Y, Thilliez G, et al (2019) Phytophthora infestans RXLR effectors act in concert at diverse subcellular locations to enhance host colonization. Journal of Experimental Botany 70: $343-356$

Wang Y, Xu Y, Sun Y, Wang H, Qi J, Wan B, Ye W, Lin Y, Shao Y, Dong S, et al (2018) Leucine-rich repeat receptor-like gene screen reveals that Nicotiana RXEG1 regulates glycoside hydrolase 12 MAMP detection. Nat Commun 9: 594-12

Westerink N, Brandwagt BF, de Wit PJGM, Joosten MHAJ (2004) Cladosporium fulvum circumvents the second functional resistance gene homologue at the $C f-4$ locus (Hcr9-4E) by secretion of a stable avr4E isoform. Molecular Microbiology 54: 533-545

Win J, Krasileva KV, Kamoun S, Shirasu K, Staskawicz BJ, Banfield MJ (2012) Sequence divergent RXLR effectors share a structural fold conserved across plant pathogenic oomycete Species. PLoS Pathog 8: e1002400

Witek K, Jupe F, Witek AI, Baker D, Clark MD, Jones JDG (2016) Accelerated cloning of a potato late blight-resistance gene using RenSeq and SMRT sequencing. Nature Biotechnology 34: 656-660

Witek K, Lin X, Karki HS, Jupe F, Witek AI, Steuernagel B, Stam R, van Oosterhout C, Fairhead S, Heal R, et al (2021) A complex resistance locus in Solanum americanum recognizes a conserved Phytophthora effector. NPLANTS 7: 198-208

Wu C-H, Abd-El-Haliem A, Bozkur TO, Belhaj K, Terauchi R, Vossen JH, Kamoun S (2017) NLR network mediates immunity to diverse plant pathogens. Proceedings of the National Academy of Sciences 114: 8113-8118

Wu C-H, Adachi H, la Concepcion De JC, Castells-Graells R, Nekrasov V, Kamoun S (2020) NRC4 Gene cluster Is not essential for bacterial flagellin-triggered immunity. PLANT PHYSIOLOGY 182: 455-459

Zhu M, Jiang L, Bai B, Zhao W, Chen X, Li J, Liu Y, Chen Z, Wang B, Wang C, et al (2017) The intracellular immune receptor Sw-5b confers broad-spectrum resistance to tospoviruses through recognition of a conserved 21 -amino acid viral effector epitope. The Plant Cell 29: 2214-2232 
bioRxiv preprint doi: https://doi.org/10.1101/2021.06.10.447899; this version posted June 10, 2021. The copyright holder for this preprint

(which was not certified by peer review) is the author/funder, who has granted bioRxiv a license to display the preprint in perpetuity. It is made available under aCC-BY-ND 4.0 International license. mediated transformation. Cell 125: 749-760 


\section{Acknowledgements}

566 This research was financed from BBSRC grant BB/P021646/1 and the Gatsby Charitable

567 Foundation. We thank TSL transformation team (Matthew Smoker and Jodie Taylor), SynBio

568 team (Mark Youles) and horticultural team (Sara Perkins, Justine Smith, Lesley Phillips and

569 Catherine Taylor) for their support. We thank Experimental Garden and Genebank of Radboud

570 University, Nijmegen, The Netherlands, IPK Gatersleben, Germany and Sandra Knapp

571 (Natural History Museum, London, UK) for access to S. americanum and S. nigrum genetic

572 diversity. We thank He Meng and Lirui Cheng from CAAS for kindly sharing the Phytophthora

573 parasitica isolates $\mathrm{R} 0$ and $\mathrm{R} 1$, and Franck Panabières from INRA for kindly sharing the

574 Phytophthora parasitica isolates 310, 329, 666 and 721. We thank Joe Win from TSL for

575 maintaining the P. palmivora strains. We thank Paul Birch and colleagues at James Hutton

576 Institute for making available clones of some of the effectors that were tested for AVRamr3

577 function.

578

579 Author contributions:

580 X.L. and J.D.G.J. designed the study. X.L., A.C.O.A., R.H., K.W., H.S.K., T.S., C.-H.W. and

581 H.A. performed the experiments. X.L., A.C.O.A., R.H. and K.W. analysed the data. X.L. and

582 J.D.G.J. wrote the manuscript with input from all authors. S.K. and V.G.A.A.V. contributed resources. All authors approved the manuscript.

584

\section{Conflict of interest:}

586 K. W. and J.D.G.J. are named inventors on a patent application (PCT/US2016/031119)

587 pertaining to Rpi-amr3 that was filed by the 2Blades Foundation on behalf of the Sainsbury

588 Laboratory. The other authors declare no competing interests. 
bioRxiv preprint doi: $h t t p s: / / d o i . o r g / 10.1101 / 2021.06 .10 .447899 ;$ this version posted June 10, 2021. The copyright holder for this preprint (which was not certified by peer review) is the author/funder, who has granted bioRxiv a license to display the preprint in perpetuity. It is made available under aCC-BY-ND 4.0 International license.

593 Supplementary files:

594
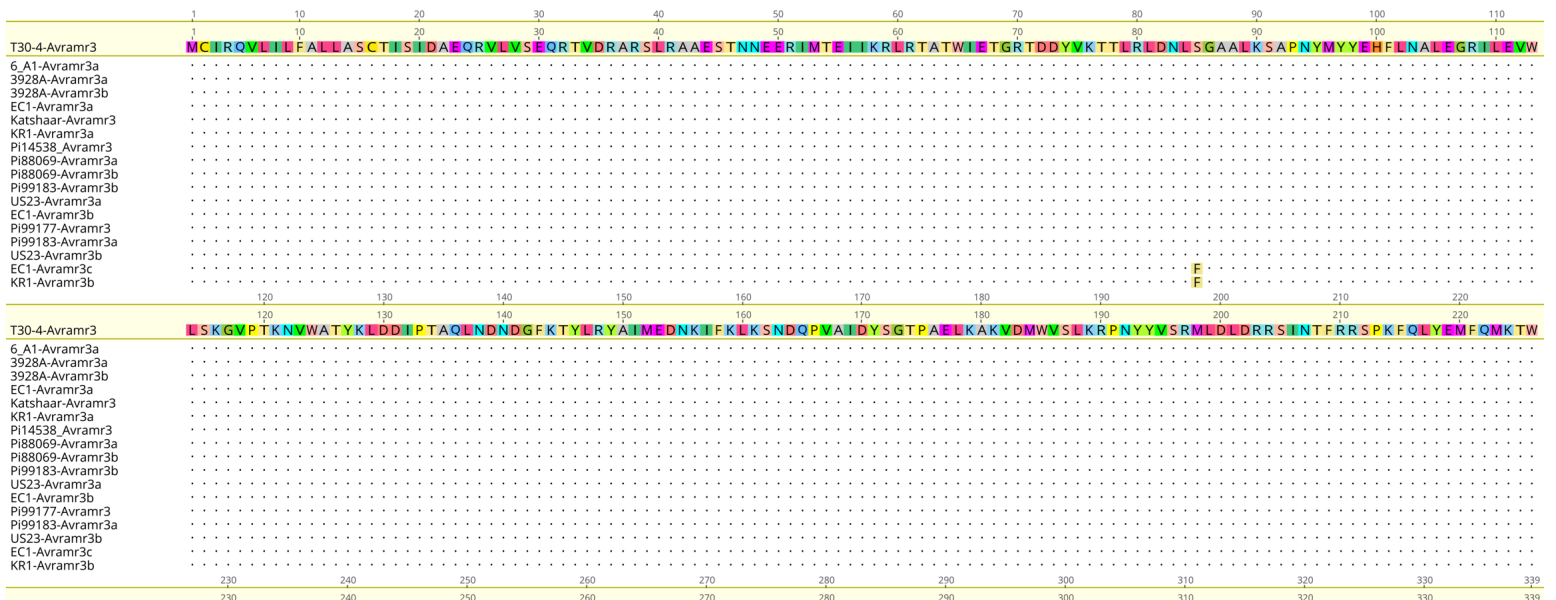

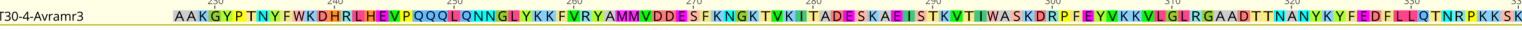
5.A1-Avramr3a

596 Figure S1: Protein alignment reveals strong conservation of $P$. infestans AVRamr3 alleles and

597 paralogs 


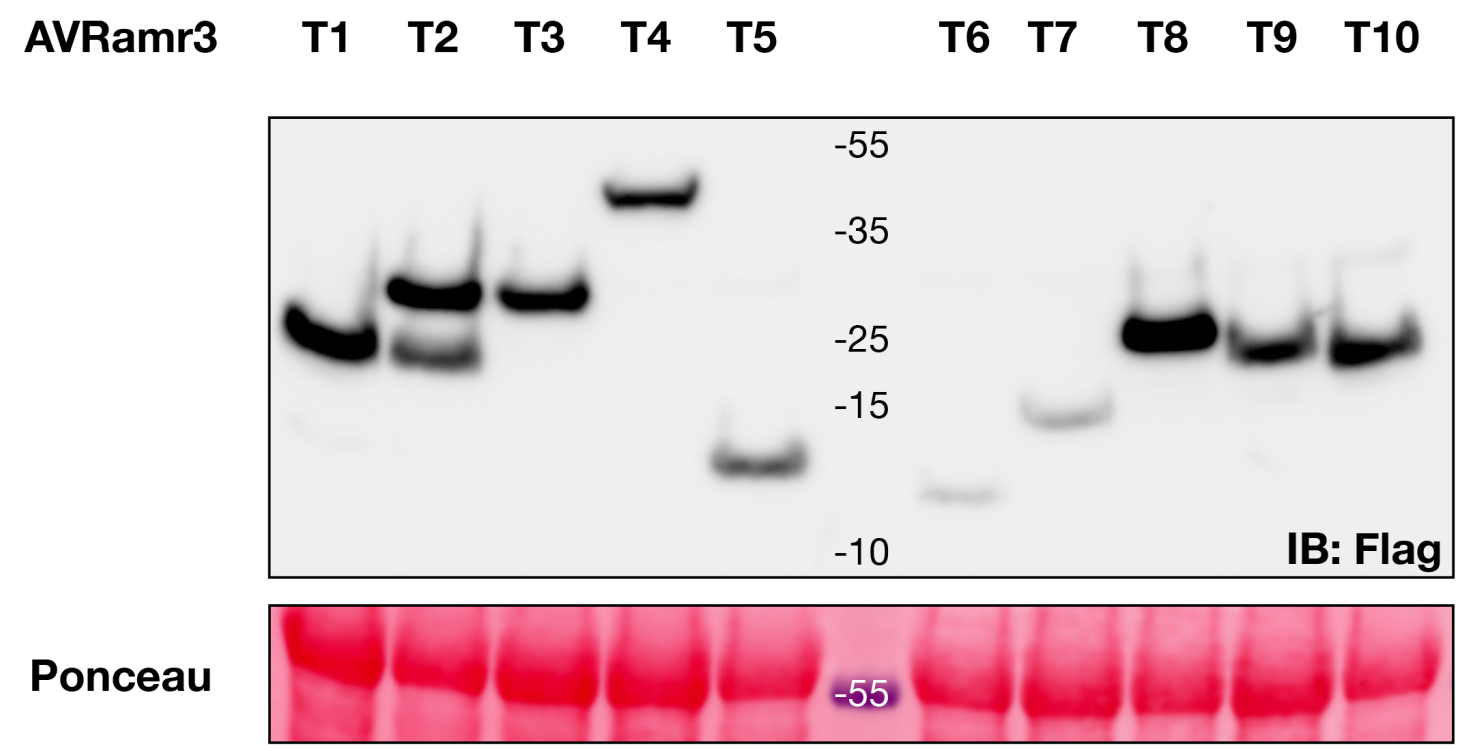

598

599 Figure S2: Western blot for AVRamr3 truncations with C-terminus HIS-FLAG tag.

600 
601

a
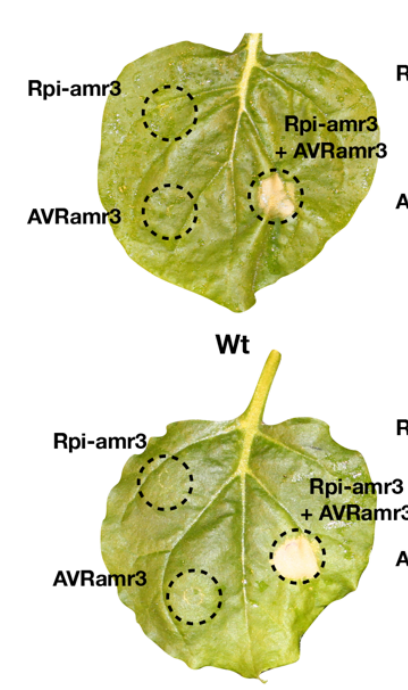

nrc4_185.9.1.3

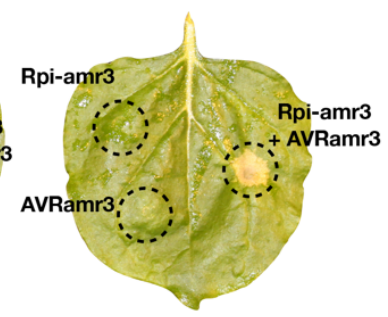

nrc2/3_1.3.1

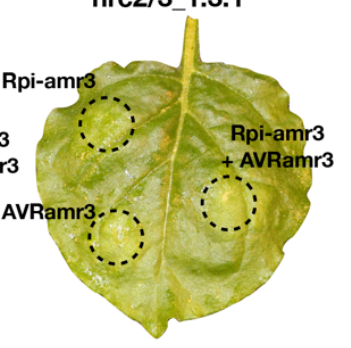

nrc2/3/4_210.4.3

Agro infiltration

b
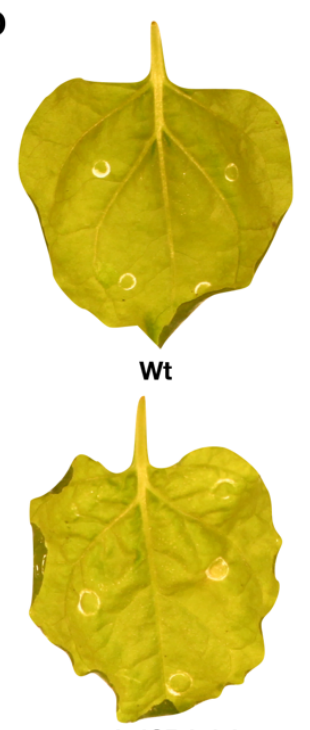

nrc4_185.9.1.3

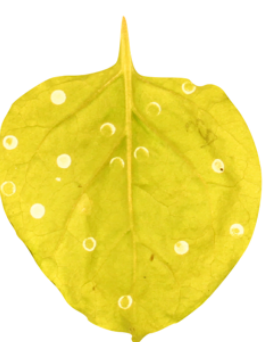

nrc2/3_1.3.1

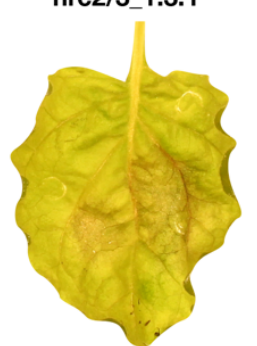

nrc2/3/4_210.4.3

Agro-infiltration of 35S::Rpi-amr3 $+P$. infestans 88069, 8dpi

602

603 Figure S3: Rpi-amr3 is NRC2, NRC3 or NRC4 dependent. (a) HR phenotype after expressing

604 Rpi-amr3, AVRamr3 and Rpi-amr3+AVRamr3 in wild type, nrc2/3_1.3.1, nrc4_185.9.1.3 and

605 nrc2/3/4_210.4.3 knockout $N$. benthamiana lines. (b) Detached leaf assay (DLA) after Rpi-

$606 a m r 3$ transient expression in wild type, nrc2/3_1.3.1, nrc4_185.9.1.3 and nrc2/3/4_210.4.3

607 knockout $N$. benthamiana. 500 zoopores of $P$. infestans 88069 were used one day after Rpi-

608 amr3 transient expression by agro-infiltration. The photos were taken eight days after 609 inoculation.

610 


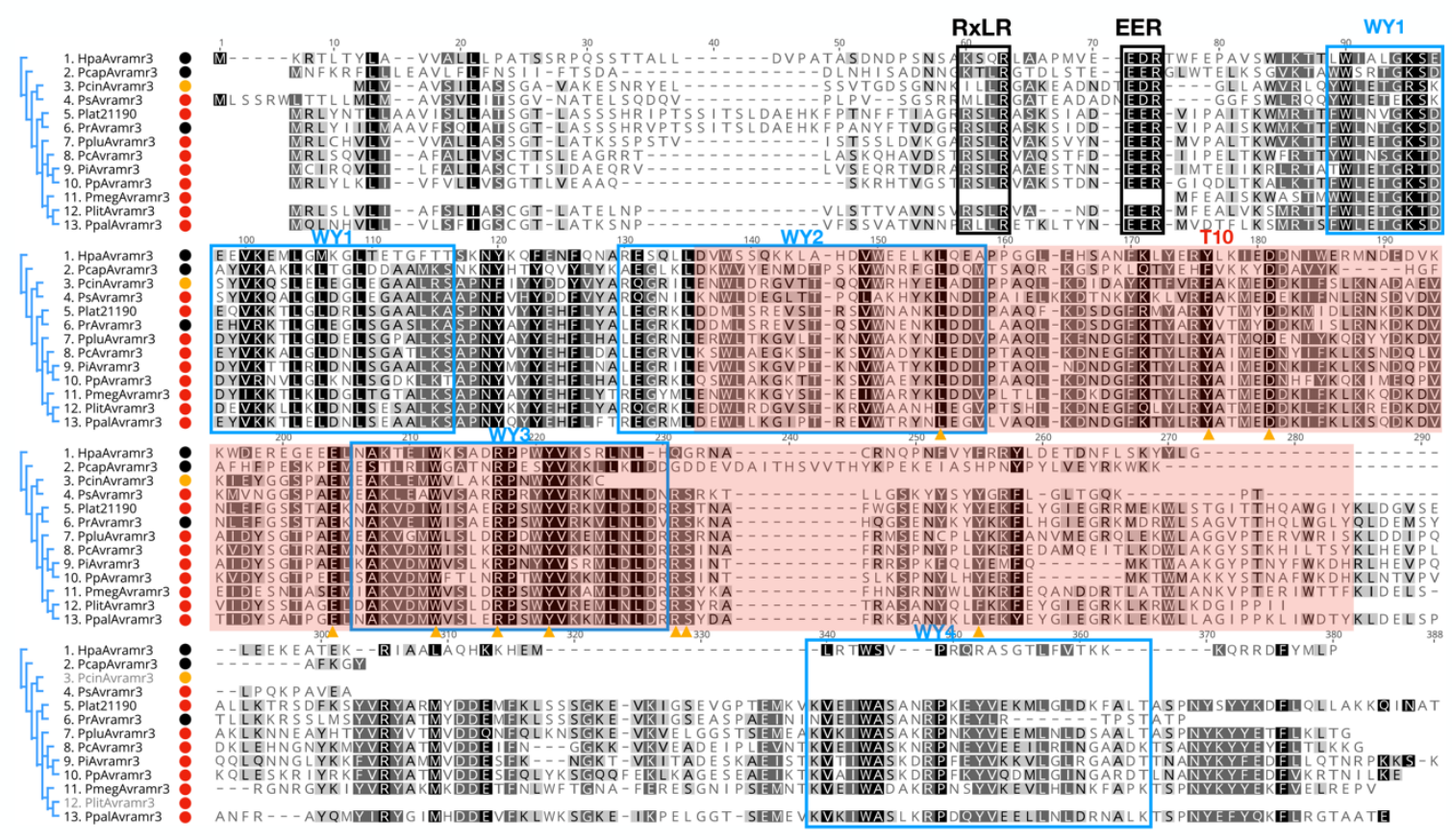

612 Figure S4: Protein alignment of AVRamr3 homologs from different Phytophthora genomes,

613 including Phytophthora infestans (Pi), Phytophthora parasitica (Pp), Phytophthora cactorum

614 (Pc), Phytophthora palmivora (Ppal), Phytophthora megakarya (Pmeg), Phytophthora litchi

615 (Plit), Phytophthora sojae (Ps), Phytophthora lateralis (Plat), Phytophthora pluvialis (Pplu),

616 Phytophthora ramorum (Pr), P. cinnamomi (Pcin), P. capsica (Pcap) and Hyaloperonospora

617 arabidopsidis (Hpa). The circles after the name are their recognition specificity by Rpi-amr3

618 in the HR assay, red: HR; black: no HR; yellow: weak HR. The RXLR and EER motifs are

619 marked by black boxes. The predicted WY motifs are marked by blue boxes. The conserved 620 amino acids which were selected for mutagenesis (see Figure S5) are marked by yellow arrows. 


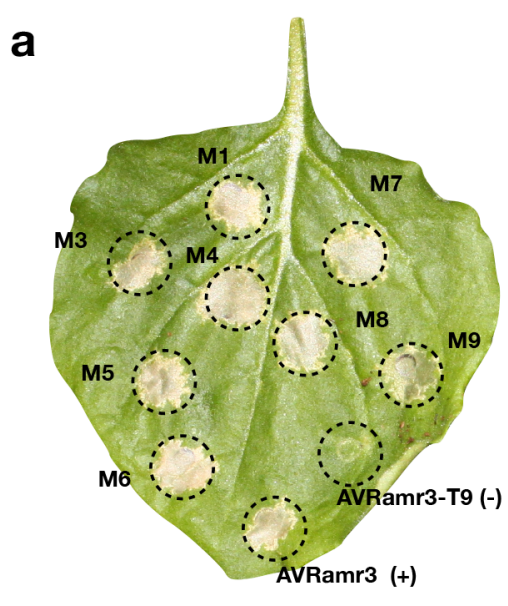

+ Rpi-amr3

\section{b}

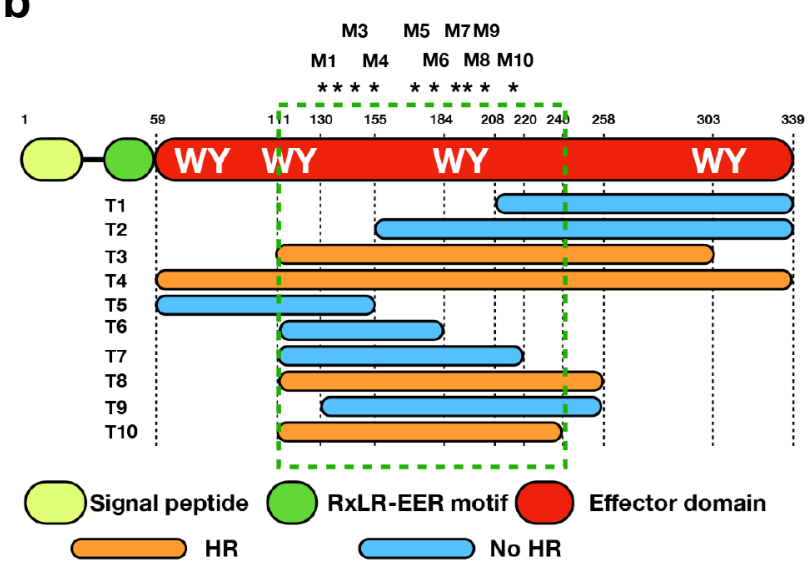

623

624 Figure S5: Mutagenesis of AVRamr3 from Phytophthora infestans. (a) Eight Avramr3

625 mutants were generated by PCR and cloned into over-expression vector with $35 \mathrm{~S}$ promoter.

626 All of them induce HR when co-expressed with Rpi-amr3. An AVRamr3 truncation T9 was

627 used as negative control, full-length AVRamr3 was used as positive control. (b) The position

628 of each mutation is marked by asterisk, and correspond to those amino acids marked by yellow

629 arrows in Fig S4. All the mutants are in the T10 region and are conserved among different

630 AVRamr3 homologs from other Phytophthora species (see Figure S4).

631 
bioRxiv preprint doi: https://doi.org/10.1101/2021.06.10.447899; this version posted June 10, 2021. The copyright holder for this preprint (which was not certified by peer review) is the author/funder, who has granted bioRxiv a license to display the preprint in perpetuity. It is made available under aCC-BY-ND 4.0 International license.

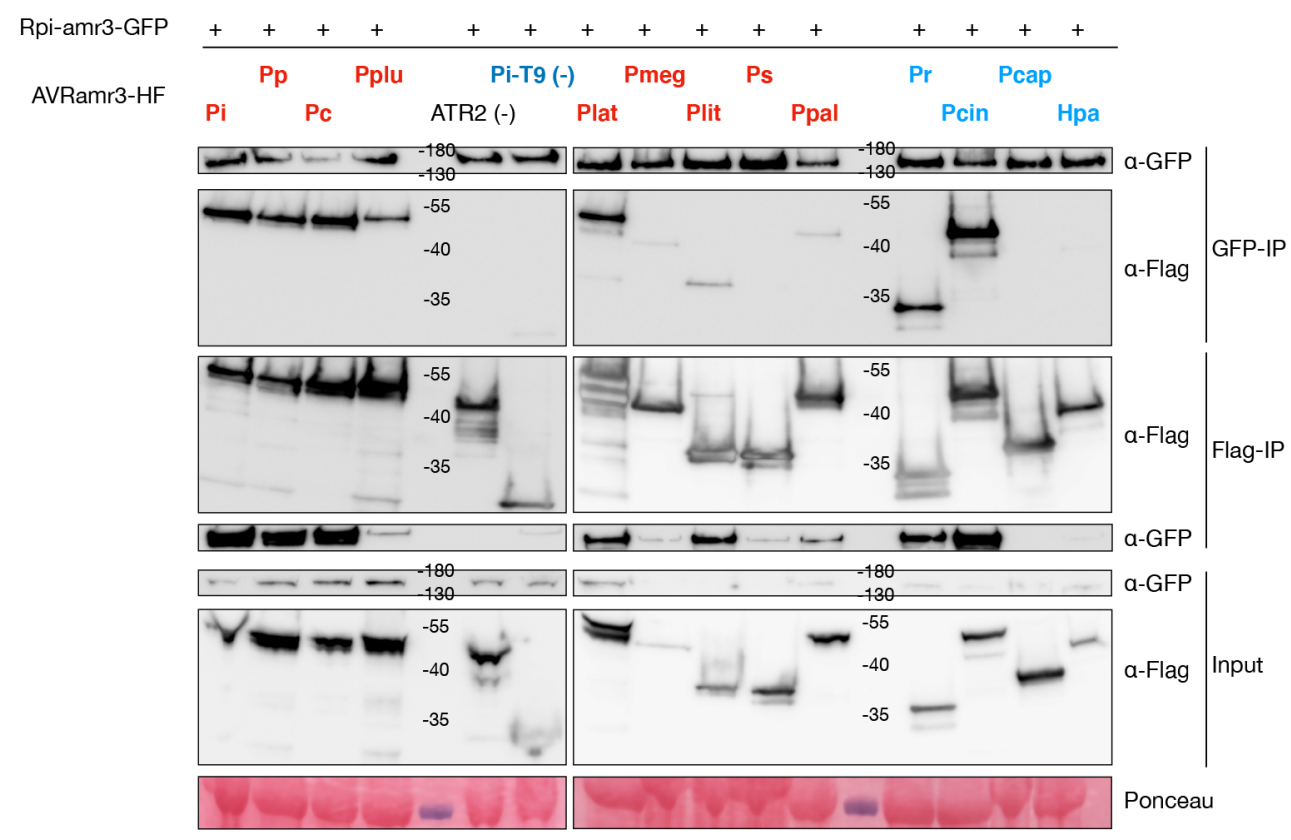

632

633 Figure S6: Co-IP of Rpi-amr3 and AVRamr3 homologs. Rpi-amr3 is tagged with C-terminal 634 GFP, and all AVRamr3 homologs are fused with a C-terminal HIS-FLAG tag. Bi-directional 635 Co-IPs were performed by GFP-IP or Flag-IP individually then incubated with Flag-HRP or 636 GFP-HRP antibody. ATR2 and AVRamr3 truncation T9 were used as negative controls. 
a

Rpi-amr3-Cluc

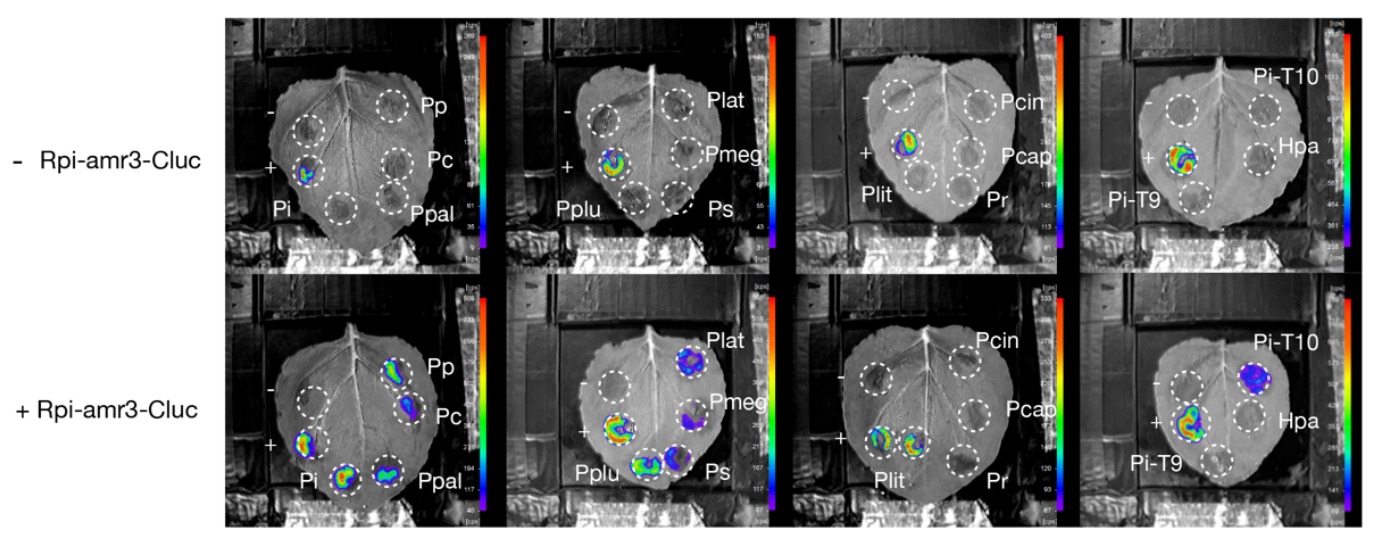

b

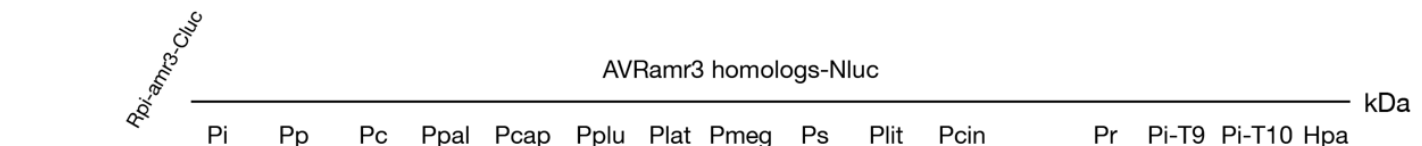

639 Figure S7: Split luciferase for Rpi-amr3 and AVRamr3 homologs from different Phytophthora

640 species. Rpi-amr3 was fused with Flag-Cluc and AVRamr3 homologs were fused with Flag-

641 Nluc. The experiment was performed in nrc2/3/4_210.4.3 knockout Nicotiana benthamiana to

642 abolish HR. (a). Expressing the AVRamr3::Flag-Nluc homologs alone does not show

643 luciferase signal; Co-expression of Rpi-amr3::Flag-Cluc with AVRamr3::Flag-Nluc homologs

644 can induce luciferase signal, specifically, P. infestans (Pi), P. parasitica $(\mathrm{Pp})$, P. cactorum $(\mathrm{Pc})$,

645 P. palmivora (Ppal), P. megakarya (Pmeg), P. litchi (Plit), P. sojae (Ps), P. lateralis (Plat) and

646 P. pluvialis (Pplu) interact with Rpi-amr3::Flag-Cluc; AVRamr3 homologs from P. ramorum

647 (Pr), P. cinnamomi (Pcin), P. capsici (Pcap) and Hyaloperonospora arabidopsidis (Hpa) do

648 not interact with Rpi-amr3 in this assay. (b). Western blot by FLAG antibody was performed

649 to confirm the expression of all proteins.

650 
bioRxiv preprint doi: $h t t p s: / / d o i . o r g / 10.1101 / 2021.06 .10 .447899 ;$ this version posted June 10, 2021. The copyright holder for this preprint (which was not certified by peer review) is the author/funder, who has granted bioRxiv a license to display the preprint in perpetuity. It is made available under aCC-BY-ND 4.0 International license.

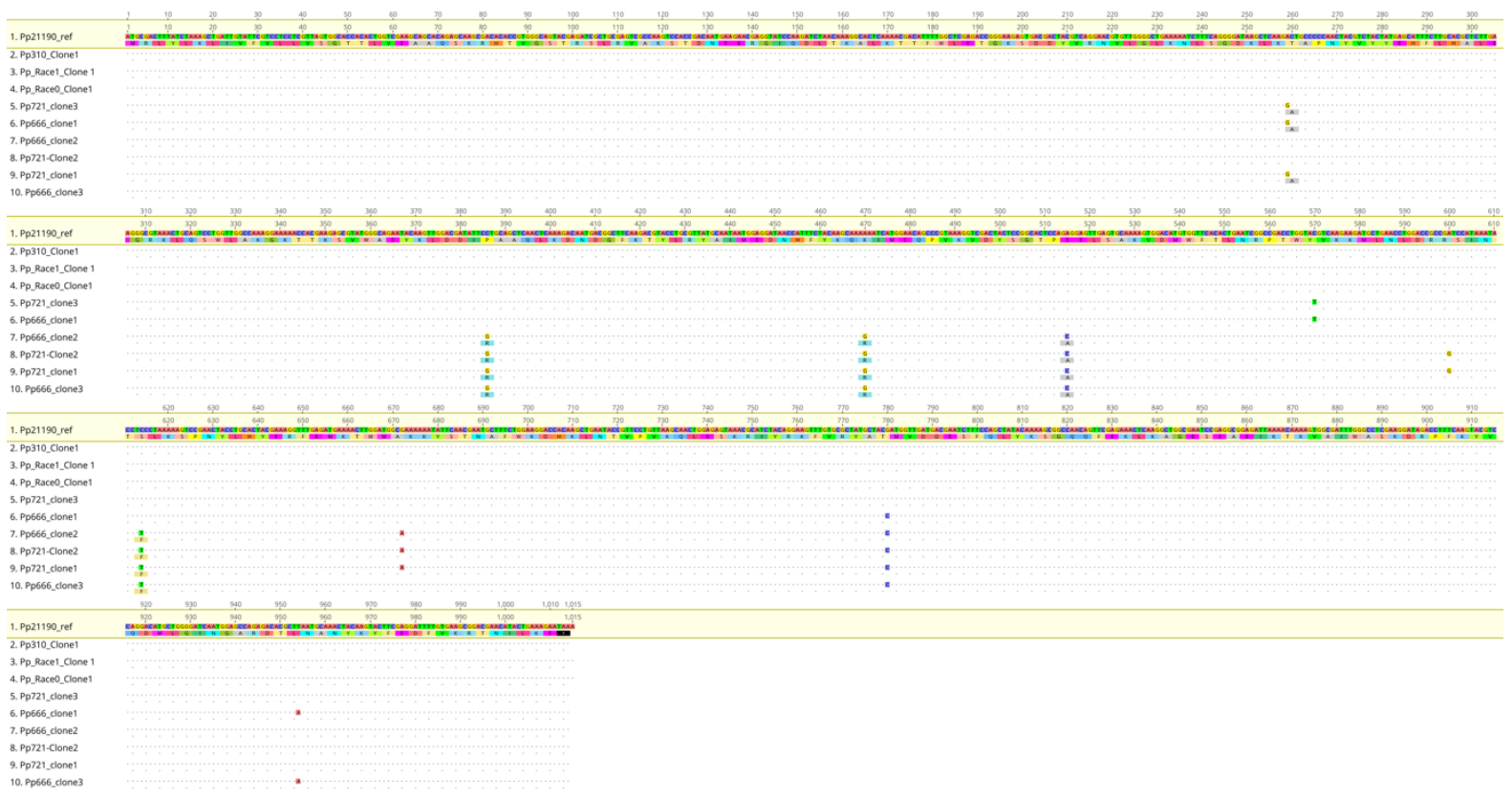

652 Figure S8: DNA alignment of Avramr3 homologs from different Phytophthora parasitica 653 isolates. The polymorphic DNA and amino acids are highlighted. 
bioRxiv preprint doi: https://doi.org/10.1101/2021.06.10.447899; this version posted June 10, 2021. The copyright holder for this preprint (which was not certified by peer review) is the author/funder, who has granted bioRxiv a license to display the preprint in perpetuity. It is made available under aCC-BY-ND 4.0 International license.

655

656

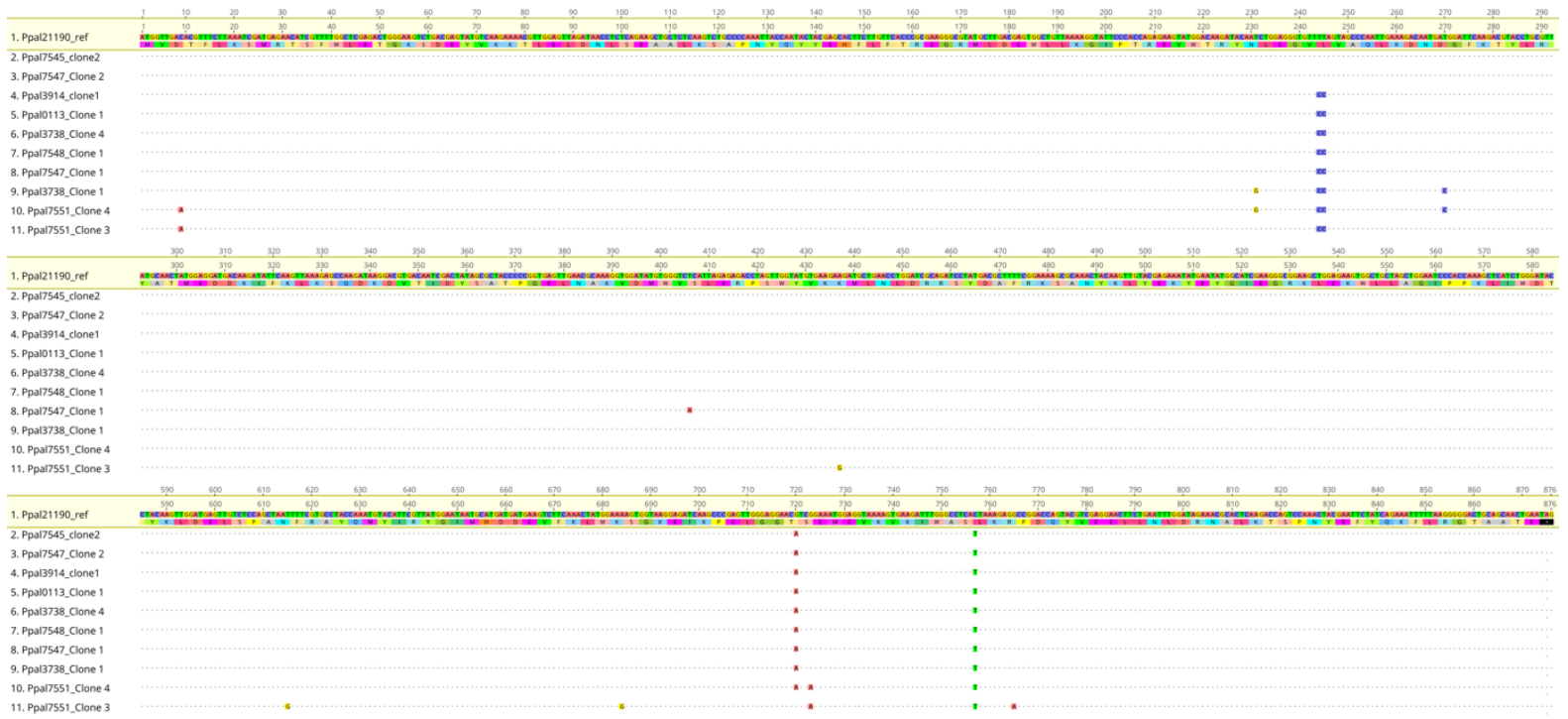

657 Figure S9: DNA alignment of Avramr3 homologs from different Phytophthora palmivora 658 isolates. The polymorphic DNA and amino acids are highlighted.

659 
bioRxiv preprint doi: https://doi.org/10.1101/2021.06.10.447899; this version posted June 10, 2021. The copyright holder for this preprint

(which was not certified by peer review) is the author/funder, who has granted bioRxiv a license to display the preprint in perpetuity. It is made available under aCC-BY-ND 4.0 International license.

660

661

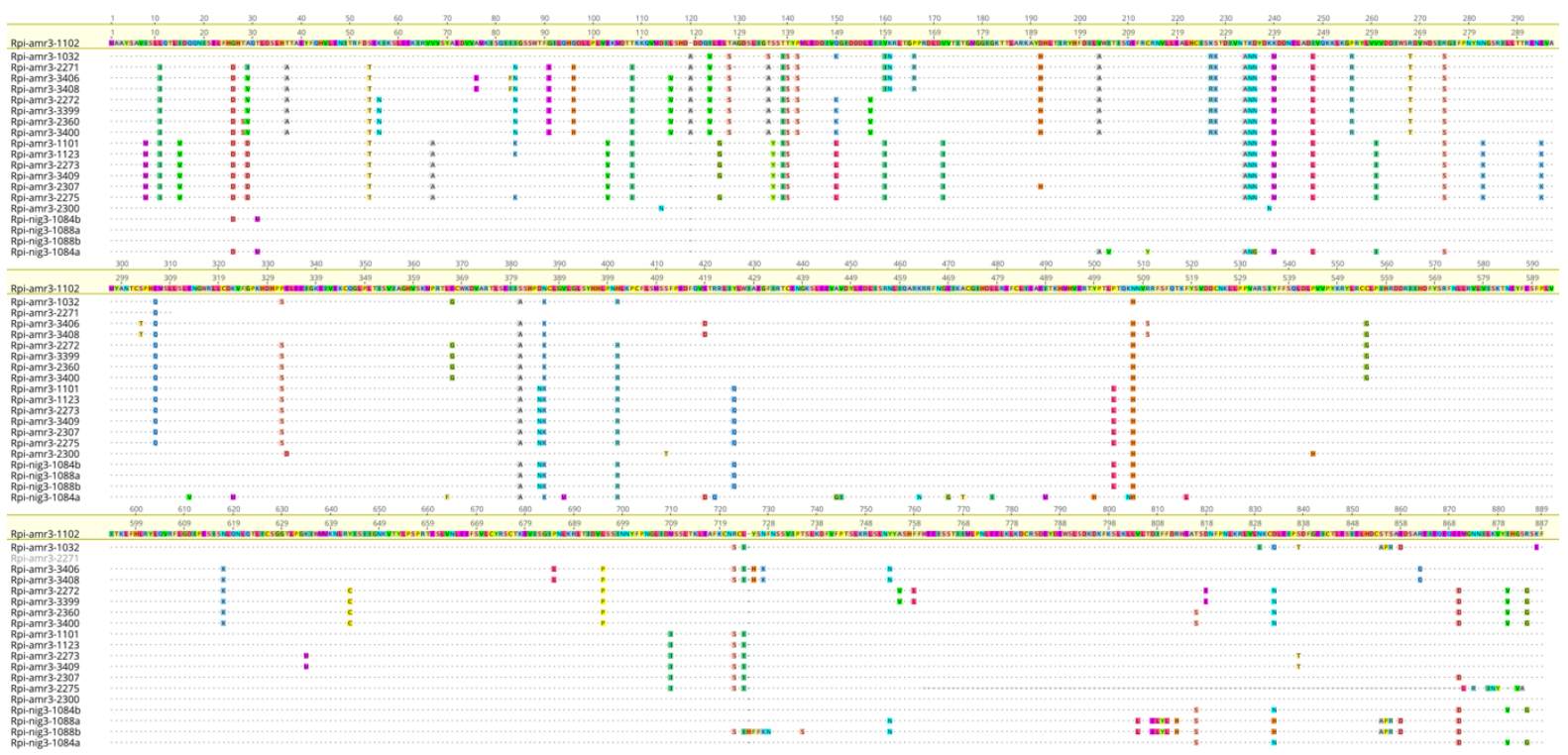

662 Figure S10: Protein alignment of Rpi-amr3 and Rpi-nig3 homologs from Solanum

663 americanum and Solanum nigrum accessions. The Rpi-amr3 was used as reference, all the 664 polymorphic amino acids are highlighted.

665

666

667

668

669 